\title{
Phytochemistry and Evidence-Based Traditional Uses of the Genus Achillea L.: An Update (2011-2021)
}

\author{
Christina Barda, Maria-Eleni Grafakou, Ekaterina-Michaela Tomou and Helen Skaltsa *(D)
}

Citation: Barda, C.; Grafakou, M.-E.; Tomou, E.-M.; Skaltsa, H.

Phytochemistry and Evidence-Based Traditional Uses of the Genus Achillea L.: An Update (2011-2021). Sci.

Pharm. 2021, 89, 50.

https://doi.org/10.3390/

scipharm 89040050

Academic Editor: Roman B. Lesyk

Received: 24 October 2021

Accepted: 18 November 2021

Published: 22 November 2021

Publisher's Note: MDPI stays neutral with regard to jurisdictional claims in published maps and institutional affiliations.

Copyright: (c) 2021 by the authors. Licensee MDPI, Basel, Switzerland. This article is an open access article distributed under the terms and conditions of the Creative Commons Attribution (CC BY) license (https:// creativecommons.org/licenses/by/ $4.0 /)$.
Department of Pharmacognosy \& Chemistry of Natural Products, Faculty of Pharmacy, School of Health Sciences, National \& Kapodistrian University of Athens, Panepistimiopolis, Zografou, 15771 Athens, Greece; cbarda@pharm.uoa.gr (C.B.); megrafakou@pharm.uoa.gr (M.-E.G.); ktomou@pharm.uoa.gr (E.-M.T.)

* Correspondence: skaltsa@pharm.uoa.gr; Tel.: +30-2107274593

\begin{abstract}
Knowledge within the field of phytochemistry research has accelerated at a tremendous speed. The excess of literature reports featuring plants of high ethnopharmacological importance, in combination with our interest in the Asteraceae family and traditional medicine, led us to acknowledge the value of the Achillea L. genus. In a broad context, the various Achillea species are used around the globe for the prevention and treatment of different diseases, including gastrointestinal problems, haemorrhages, pneumonia, rheumatic pains, diuresis, inflammation, infections, and wounds, as well as menstrual and gynaecologic abnormalities. The present review aims to provide and summarize the recent literature (2011-2021) on the phytochemistry of the Achillea genus. In parallel, this study attempts to bridge the reports on the traditional uses with modern pharmacological data. Research articles that focused on secondary metabolites, traditional uses and pharmacological activities were collected from various scientific databases such as Pubmed, ScienceDirect, Reaxys and Google Scholar. This study revealed the presence of 141 phytochemicals, while 24 traditionally used Achillea spp. were discussed in comparison to current data with an experimental basis.
\end{abstract}

Keywords: Achillea spp.; phytochemicals; flavonoids; sesquiterpene lactones; pharmacological activities; traditional uses

\section{Introduction}

The traditional knowledge of medicinal plants is a cornerstone on which medicine has been built since the beginning of mankind [1]. By combining this traditional knowledge with new methods derived from modern science, the possibilities for drug development seem endless. Thus, the field of ethnopharmacology has expanded greatly, with a plethora of articles published each year.

The excess of literature reports featuring plants of high ethnopharmacological importance, in combination with our interest in the Asteraceae family and traditional medicine, led us to acknowledge the value of the Achillea L. genus. The genus Achillea (Asteraceae, Anthemideae) comprises over 130 perennial and flowering species, which are mainly distributed in the northern hemisphere with diversity centres in S.E. Europe and S.W. Asia [2,3]. Remarkably, the genus is characterized by its ability to adapt in diverse ecological amplitudes and ranges in extreme environmental conditions, such as deserts to water-logged habitats and from seacoasts to the nival zone of high mountains or mild agricultural areas [4]. The most obvious and outstanding characteristics of this genus are the numerous flowers, small and white, yellow, orange, pink or red. Specifically, the genus can be described as perennial herbs of $6-80 \mathrm{~cm}$ tall with alternate leaves and finely pinnatisect. The capitula in corymbs are heterogamous with seriate phyllaries. The achenes are strongly flattened and not ribbed and the fruits are shiny, with no pappus [5]. The name of the genus derives from the Greek hero, Achilles. According to Greek mythology, this powerful warrior was tutored by the centaur Chiron in the arts of war, music, and medicine. During the Trojan War, Achilles used Achillea, whose virtues for blood control and wound 
healing were well-known, to treat his fellow soldier Telephos. Historically, the medicinal properties of these plants are described in the ancient medical texts of Dioscorides (1st c.A.D.) and Pliny the elder (1st c.A.D.) [6,7].

Several species of this genus are widely used in traditional medicines of different countries. In particular, A. millefolium aggr. (closely related species that are treated as a single species), known as common yarrow, is utilized in crude forms for various therapeutic purposes such as teas for digestive problems and respiratory infections or poultices powders for skin disorders, etc. [8]. Nowadays, monographs by the WHO (World Health Organization; www.who.int (accessed on 1 September 2021)) [9] and EMA (European Medicines Agency; www.ema.europa.eu (accessed on 1 September 2021)) [10] also address the medicinal use of $A$. millefolium $\mathrm{L}$. for loss of appetite, skin disorders and minor wounds, urinary tract, genital and gastrointestinal disorders. Plenty of research studies are conducted to determine the biological activities of this genus, focusing not only on crude forms and extracts but also on isolated specialized products extracted in solvents of different polarities.

Some of the reported and validated pharmacological activities (in vitro, in vivo, and clinical tests) of the Achillea species are antioxidant, anti-inflammatory, analgesic, antipyretic, antidiabetic, antibacterial, anthelmintic, antihypertensive. These important biological effects are directly correlated to plant-derived bio-active phytochemicals. Previous reports demonstrated the occurrence of various chemical constituents in Achillea species, with sesquiterpene lactones and flavonoids as the principal bioactive constituents. Moreover, triterpenes, sterols, coumarins and phenolic acids were also reported [11]. Additionally, there are a lot of studies reporting the volatile constituents of the genus, which have been summarized in recent years [12,13]. Essential oils, isolated from Achillea spp., as secondary metabolites involve complex mixtures that also contribute significantly to the observed biological activities against a variety of ailments, and especially intestinal disorders and inflammatory diseases [12,13].

A landmark study by $\mathrm{Si}$ et al. [11] was meticulously planned, including the structures and biological properties of known phytochemicals from Achillea spp., followed by the comprehensive summary of Saeidnia et al. [14] and other numerous reviews on this topic $[12,13]$. Therefore, ten years after the last overviews by Si et al. [11] and Saeidnia et al. [14] (2006 and 2011, respectively), the present review aims to provide and summarize the existing knowledge on the genus Achillea, focusing on traditional uses, phytochemistry and pharmacological activities. In comparison with a similar study [12] in our review, we exhaustively summarized the non-volatile secondary metabolites of the last decade, identifying more than 141 chemical entities whose structures are depicted herein. Moreover, this overview gives an account of the ethnopharmacological relevance for the traditional uses of the genus supported by modern pharmacological research knowledge. Specifically, aspects of the folkloric uses of this genus are discussed in comparison to current data with an experimental basis. It is hoped that such work could encourage researchers to continue working on this outstanding genus, aiming to unveil the whole potential of its diverse phytochemical profile, with flavonoids and sesquiterpene lactones being lead compounds for future drug development, targeting serious human ailments.

\section{Materials and Methods}

A comprehensive search on the relevant literature of the last decade was conducted on the major scientific databases, including Pubmed, ScienceDirect, Reaxys and Google Scholar. The search terms "Achillea", "Achillea genus", "Achillea species", "Achillea medicinal uses", "Achillea pharmacological", "Achillea traditional uses", "Achillea compounds", "Achillea phytochemicals", "Achillea biological" and "Achillea bioassays", were used for data collection. We are also aware of the fact that these databases are updated routinely; thus, the data presented in Table 1a are indicative of the available information at the time this paper was prepared. Searches were also performed for other potential studies by manual screening references in the identified studies. 
Table 1. (a) Bibliometric results of keyword/phrase search for genus Achillea. (b) Bibliometric results of the top 5: co-occurring authors, cited documents and keywords.

\begin{tabular}{|c|c|c|c|c|}
\hline \multicolumn{5}{|c|}{ a } \\
\hline \multirow[t]{2}{*}{ Keyword/Phrase } & \multicolumn{4}{|c|}{ Number of Hits } \\
\hline & PubMed & ScienceDirect & Reaxys & Google Scholar \\
\hline Achillea & 384 & 2.238 & 1.819 & 19.700 \\
\hline Achillea genus & 21 & 558 & 73 & 14.400 \\
\hline Achillea species & 150 & 1.678 & 678 & 17.000 \\
\hline Achillea medicinal uses & 25 & 857 & 616 & 13.000 \\
\hline $\begin{array}{c}\text { Achillea } \\
\text { pharmacological }\end{array}$ & 169 & 517 & 98 & 8.600 \\
\hline Achillea traditional uses & 21 & 956 & 62 & 15.500 \\
\hline Achillea compounds & 122 & 1.091 & 750 & 14.300 \\
\hline Achillea phytochemicals & 39 & 437 & 103 & 7.110 \\
\hline Achillea biological & 249 & 1.075 & 193 & 16.900 \\
\hline Achillea bioassays & 25 & 144 & 20 & 2.560 \\
\hline \multicolumn{5}{|c|}{$\mathbf{b}$} \\
\hline \multicolumn{2}{|c|}{ Most co-occurred authors } & \multicolumn{3}{|c|}{$\begin{array}{l}\text { Formari Tiziana; Amsilinger Sabine; Venditti } \\
\text { Alessandro; Akram Muhammad; Skaltsa Helen }\end{array}$} \\
\hline \multicolumn{2}{|c|}{ Most cited documents } & \multicolumn{3}{|c|}{$\begin{array}{c}\text { Saednia et al., 2011; Vitalini et al., 2011; } \\
\text { Mohammadhosseini et al., 2017; Baretta, et al., 2012; } \\
\text { Agar et al., } 2015\end{array}$} \\
\hline \multicolumn{2}{|c|}{ Most used keywords } & \multicolumn{3}{|c|}{$\begin{array}{c}\text { Achillea/Chemistry, A. milefolium, plan } \\
\text { extracts/pharmacology, phytotherapy, medicinal } \\
\text { plants }\end{array}$} \\
\hline
\end{tabular}

A bibliometric method was applied using VOSviewer (version 1.6.15) in "Full Record and Cited References" for further analysis under the following parameters: co-authorship, co-occurrence, citation, bibliographic coupling and themes. The analysed articles were retrieved from the PubMed database. Map data visualizations were generated to build (a) a keyword map based on shared networks, (b) a term map from the titles and abstracts on shared networks, and (c) the co-authorship/co-occurrence authors network (see Supplementary Figures S1s, S2 and S3). The top 5 most cited authors, documents and keywords are provided in Table $1 \mathrm{~b}$.

For the selection of relevant publications, three major criteria were used in this review:

A. Phytochemicals: For the chemical compounds, all the publications that could be accessed were included with any information on the isolation or/and identification methods, excluding articles solely on essential oil and volatile constituents.

B. Ethnopharmacology: For the traditional uses all the publications that could be accessed were included with any information on local medicinal uses of the genus Achillea.

C. Pharmacological activities: For this section, only the literature data which associate the traditional knowledge of the genus Achillea with the pharmacological activities were included.

Members of the genus Achillea are presented as individual species in this work and for the validation of their scientific names the International Plant Name Index (www.ipni. org (accessed on 1 September 2021)), the Plant list (www.theplantlist.org (accessed on 1 November 2021)) and/or the Euro+Med (www.emplantbase.org (1 September 2021)) databases were used. This helped to identify misspellings and the use of synonyms for different or related species.

Order of covered compounds: Due to the reticulate nature of biosynthetic pathways, there is no universal way to order natural products and compound categories. Thus, compounds covered in this review have been ordered as follows: flavonoids, phenolic acids, quinic acids, sesquiterpene lactones and other compounds. Compounds are catego- 
rized by species in Tables 2, 5 and 7, with the attempt of discrimination between publications describing metabolites by isolation or identification/screening (HPLC, LC-MS, etc.). The chemical structures of the bioactive compounds are shown in Tables 3, 4, 6 and 8-13. Finally, the traditional medicinal uses of Achillea species are reported in Table 14.

\section{Chemical Constituents}

\subsection{Phytochemicals}

Phytochemical investigations of the genus Achillea have revealed that many components are present in these plants, such as sesquiterpene lactones, mainly guaianolides and germacranolides, flavonoids, phenolic acids, coumarins, alkaloids, amino acids, fatty acids, alkanes, polyacetylenes, saponins, sterols, sugars, and essential oils [11,15]. The genus is characterized by the ability to biosynthesize terpenoids, especially sesquiterpene lactones and essential oils, being all important chemotaxonomic markers of Achillea. From a phytochemical point of view, the following section summarizes the reported phytochemicals (excluding the reports on the essential oils) in Tables 2-13.

\subsubsection{Polyphenols}

Polyphenols are one of the largest and most widespread chemical groups of natural compounds. This category of specialised products is of great interest due to their common occurrence and their attractive biological properties. Polyphenols of the genus Achillea can be divided into two sub-groups according to their chemical structures, i.e., flavonoids [16-51] and phenolic acids [16-67], based on literature data from 2011 to 2021 (Tables 2-5).

\subsubsection{Flavonoids}

Flavonoids represent the largest group of polyphenolic compounds occurring in Achillea spp. and are in accordance with those of other taxa of the Asteraceae family. In general, the pattern of these flavonoids demonstrates a tendency for methylation at the $\mathrm{C} 6$ and $\mathrm{C}^{\prime}$ positions. Furthermore, their biological activities vary depending on the structure degree of hydroxylation, substitution, and conjugation. The first isolation of flavonoids, dating back to 1961, unveiled two flavonoids with antispasmodic activity, namely cynaroside and cosmoside from A. millefolium [52-55].

Flavones and 3-hydroxyflavones are well documented by numerous studies and consist of the most frequently isolated compounds along with sesquiterpene lactones. Among them, luteolin (19), apigenin (2) and quercetin (44), with their corresponding glycosides, seem to be principal compounds in a high number of Achillea species (Table 2). Impressively, the genus accumulates a wide variety of C-glycosylflavones bearing luteolin (19) and apigenin (2) skeletons. Likewise, an array of methylated flavonoids were also obtained both as aglycons and glycosides [11,14,56]. All stated chemical categories are exhaustively discussed below.

Hereby, we report on the isolation of 71 flavonoid compounds from about 28 Achillea species (Tables 2 and 4). A total of 34 flavones have been reported, so far; among these, 26 species contain luteolin (19) and 21 apigenin (2) as aglycons (Table 3). These compounds represent simple flavonoids that widely occur in different species of this genus, as well as in Asteraceae family. Two flavones bearing a methoxy group attached to C-7 or C $-3^{\prime}$ were reported once, namely 7-O-methyl apigenin (3) (from A. ligustica) [35] and chrysoeriol (20) (from A. millefolium) [40], respectively. Moreover, other methylated flavonoids, such as cirsiliol (22) and cirsimaritin (4), were reported for A. fragrantissima [31,33]; salvigenin (5) for A. millefolium [41] and A. wilhelmsi [50]; and 8-hydroxysalvigenin (6) was identified for A. lycaonica A. monocephala and A. vermicularis [36,42,49]. Moreover, eupatorin (21) was reported for $A$. alpina and $A$. tenuifolia $[17,48]$, while eupatilin 7 -methyl ether (5-demethylsinensetin) (23) for $A$. wilhelmsii [25].

Regarding luteolin 7-O- $\beta$-D-glucoside (24), studies revealed its presence in 11 species (Tables 2 and 3), whereas luteolin 4'-O-glucoside (25) was only reported in A. alpina [19]. Apigenin 7-O-glucoside (cosmosiin) (8) was reported in A. fragrantissima and A. mille- 
folium [33,40], while apigenin 7-O-glucuronide (9) was only found in A. ligustica [45]. Furthermore, a rutinosyl derivative, namely hesperidin (29), was detected by Agar et al. [27] from A. kotschyi Boiss. subsp. kotschyi and A. lycaonica.

In addition to these regular compounds, some $C$-flavonoid ( $C$-flavones) derivatives, mainly luteolin and apigenin $C$-glucosides, were isolated from the genus Achillea. Regarding the luteolin derivatives, orientin (luteolin 8-C-glucoside) (26) was isolated from $A$. nobilis [45], while isoorientin (27), with a 6-C-glucosyl moiety, was reported from $A$. alpina, $A$. nobilis, $A$. millefolium and $A$. wilhelmsii $[17,40,45,50]$. Apigenin derivatives were reported in the form of 8-C glucoside (vitexin) (10) by Taskin et al. [45] from A. nobilis and from $A$. magnifica [37], respectively. Isovitexin (11), a 6-C-glucoside, was identified from A. alpina, A. fragrantissima, A. millefolium and A. wilhelmsii $[18,20,30,50]$. In addition, its $4^{\prime}$-methyl ether (23) was reported only in A. fragrantissima [30]. Moreover, a 7-methyl ether derivative, namely swertisin (13), was reported from $A$. wilhelmsii [50]. It is of considerable interest that the studies revealed the presence of four $C$-diglycosides: apigenin 6,8-C-diglucoside (vicenin-2) (14) in A. magnifica, A. cretica and A. wilhelmsii [37,50], apigenin 6-C-glucoside-8-C-arabinoside or schaftoside (16) from $A$. asiatica and $A$. wilhelmsii, apigenin 6-C-arabinoside-8-C-glucoside (isoschaftoside) (17) from $A$. nobilis and $A$. wilhelmsii and lastly, acacetin $6-C-\left(6^{\prime \prime}\right.$-acetyl- $\beta$-D-glucopyranoside)- $-8-C$ - $\alpha$-L-arabinopyranoside (18) from $A$. fragrantissima [31].

Furthermore, several simple flavonols (3-hydroxyflavones), either possessing methoxy groups or rarely as glycosides, were surveyed in the genus. In this study, 27 flavonols were found in the Achillea species. Among them, quercetin (44) and rutin (58) were the most representative secondary metabolites. Precisely, the first one was reported in 16 species, while the latter was found in 18 species. Another example was kaempferol (37), which was encountered in six species.

In addition, methoxylated 3-hydroxyflavones were listed, such as fisetin (35) identified in A. lycaonica [27] or 3,8-dimethylherbacetin (38) reported in A. biebersteinii [26], whereas galetin 3,6-dimethyl ether (39) was isolated from A. ligustica [3]. Likewise, some examples with low reappearance in Achillea species were quercetagetin 3,3'-dimethyl ether (48) isolated from A. millefolium [39], penduletin (40) isolated from A. alpina [18], chrysosplenol D (51) reported from A. fragrantissima [31], centaureidin (49) reported from A. millefolium [39] and $A$. coarctata [7]. Apart from the above-mentioned menthoxy- or polymethoxy-flavonols (3-hydroxyflavones), there are also others such as jaceidin (52) reported from A. alpina [18], A. biebersteinii [26] and A. millefolium [27,39]; santin (41) reported from A. biebersteinii [24] or A. ligustica [35]; casticin (53) isolated from A. coarctata [7] and A. millefolium [39]; and axillarin (47) reported from A. biebersteinii [24], A. grandifolia [45] and A. vermicularis [49]. Interestingly, Huo et al. [39] isolated for the first time a rare biflavonoid, namely 8, $8^{\prime}$-bi-3$O$-methylquercetin (70) from A. millefolium. On the other hand, only a few glycosylated flavonols derivatives were described in this genus. As mentioned above, rutin (58) appears in most Achillea species, whilst quercetin 3-O-glucoside (55) was reported in A. millefolium [40] and A. schurii [47]. Furthermore, quercetin 3-O-arabinosyl $(1 \rightarrow 6)$ glucoside (63) was reported in A. asiatica by one study [20]. Substances bearing galactose have rarely occurred in the genus; nevertheless, the 3-O-galactoside of quercetin, namely hyperoside (59), was identified in A. kotschyi Boiss. subsp. kotschyi and A. lycaonica [27]. Of note, two more compounds bearing 3-O-rutinoside were reported: a kaempferol (37) derivative in $A$. millefolium [40] and an isorhamnetin analogue (61) in A. alpina [19].

Notably, only two flavanones were mentioned, naringenin (66) in A. kotschyi Boiss. subsp. kotschyi, A. lycaonica, A. monocephala and A. vermicularis [49] and dihydroquercetin (64) from A. millefolium [41]. 
Table 2. Flavonoids from Achillea spp.

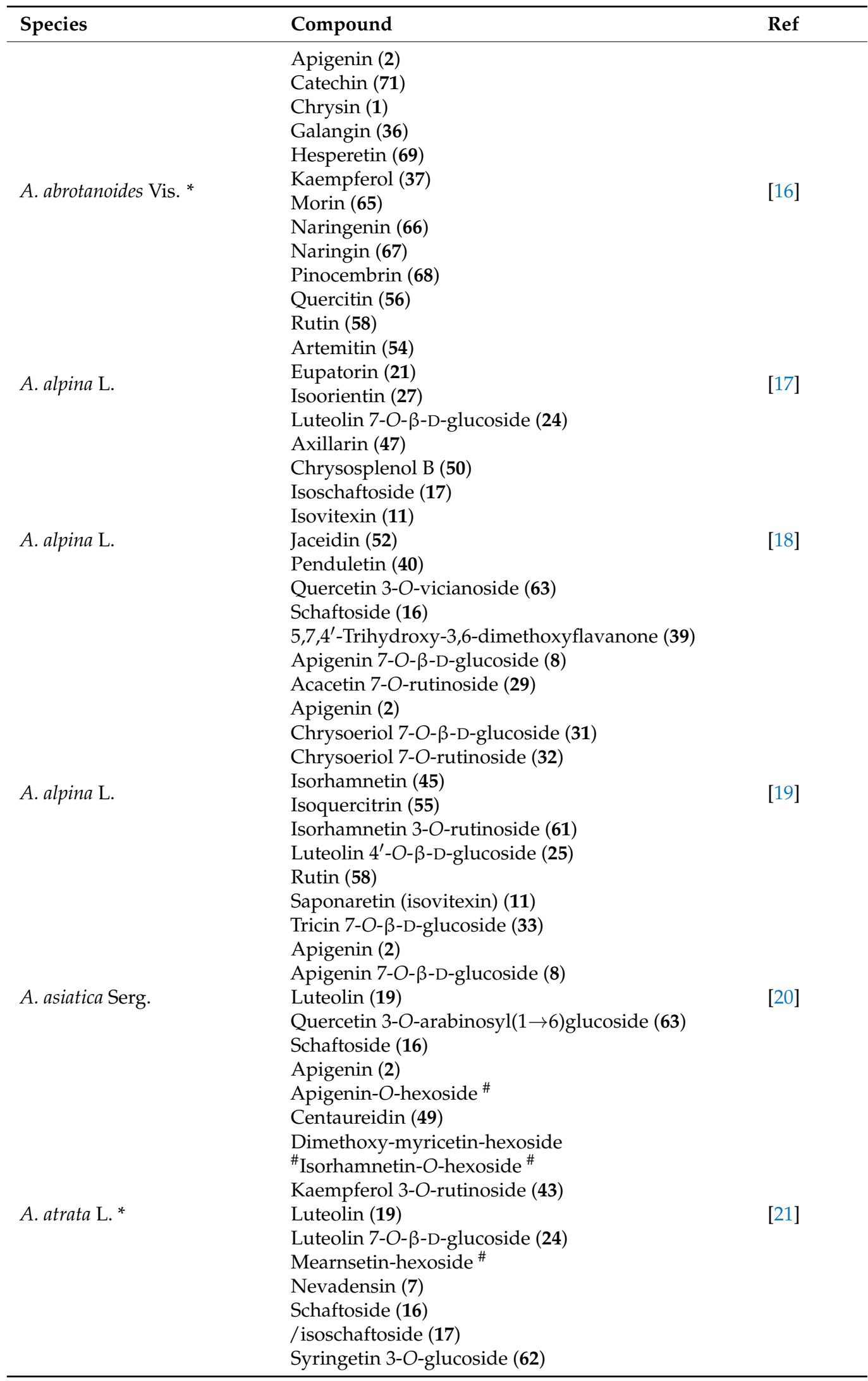


Table 2. Cont.

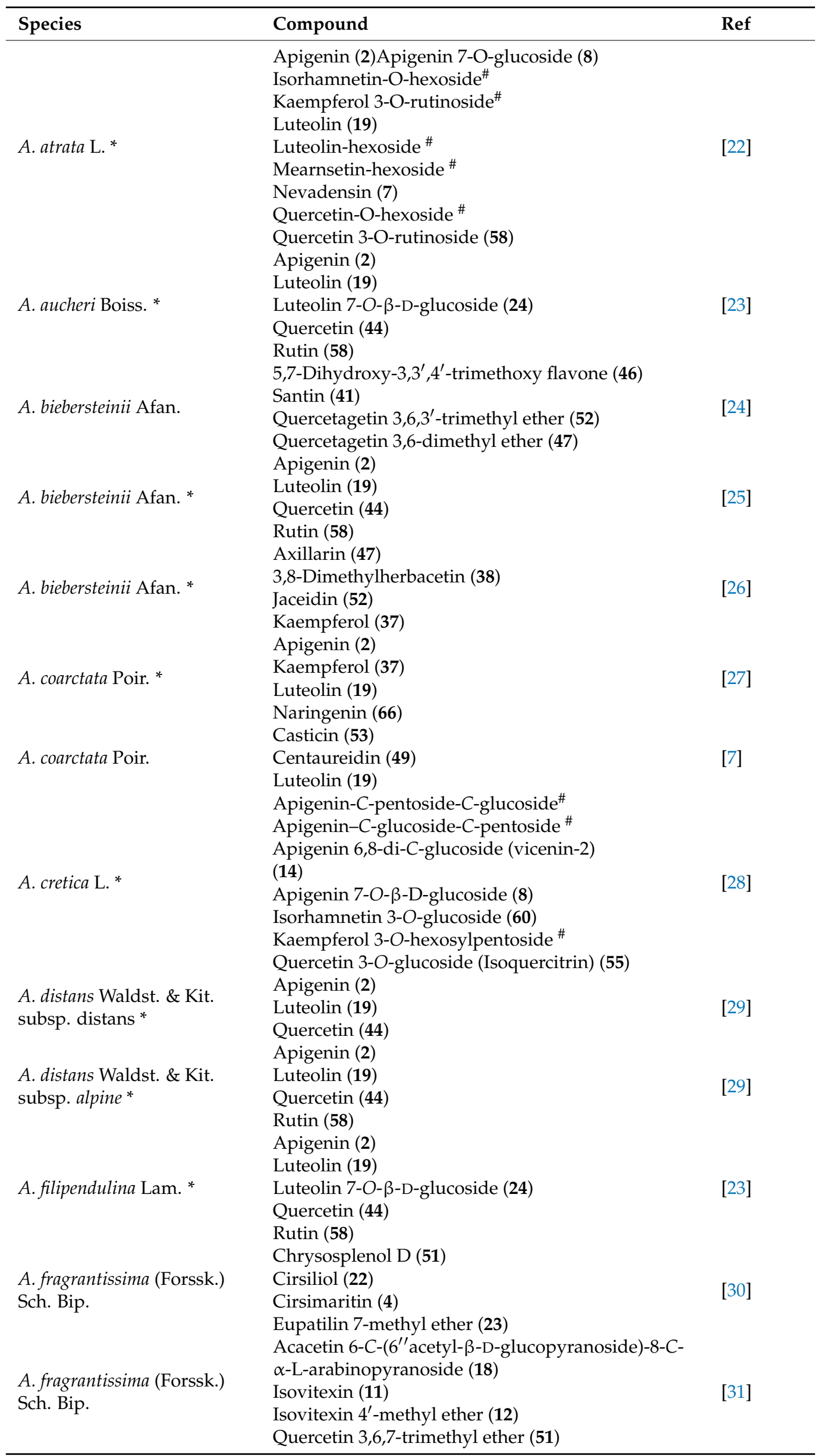


Table 2. Cont.

\begin{tabular}{|c|c|c|}
\hline Species & Compound & Ref \\
\hline A. fragrantissima (Forssk.) & Chrysosplenetine (50a) & \multirow{3}{*}{32} \\
\hline \multirow[t]{2}{*}{ Sch. Bip. } & Chrysosplenol D (51) & \\
\hline & Apigenin 6-C-glucoside (11) & \\
\hline \multirow{6}{*}{$\begin{array}{l}\text { A. fragrantissima (Forssk.) } \\
\text { Sch. Bip. }\end{array}$} & Cosmosiin (8) & \multirow{5}{*}[33]{} \\
\hline & Jaceidin (52) & \\
\hline & Luteolin (19) & \\
\hline & Quercetin 3-O-galactoside (59) & \\
\hline & Luteolin (19) & \\
\hline & Luteolin 7-O- $\beta$-D-glucoside (24) & \\
\hline \multirow[t]{6}{*}{ A. grandifolia Friv. * } & Quercetagetin 3,6-dimethyl ether (47) & \multirow[t]{6}{*}[34]{} \\
\hline & Quercetin (44) & \\
\hline & Rutin (58) & \\
\hline & Apigenin (2) & \\
\hline & Hesperidin (29) & \\
\hline & Hyperoside (59) & \\
\hline \multirow{6}{*}{$\begin{array}{l}\text { A. kotschyi Boiss. subsp. } \\
\text { kotschyi * }\end{array}$} & Kaempferol (37) & \multirow{6}{*}[27]{} \\
\hline & Luteolin (19) & \\
\hline & Naringenin (66) & \\
\hline & Quercetin (44) & \\
\hline & Rutin (58) & \\
\hline & Apigenin (2) & \\
\hline \multirow{3}{*}{ A. lingulata Waldst. } & Hesperetin (69) & \multirow{3}{*}[16]{} \\
\hline & Morin (65) & \\
\hline & Rutin (58) & \\
\hline \multirow{8}{*}{ A. ligustica All. } & 7-O-methyl apigenin (3) & \multirow{8}{*}{35} \\
\hline & Apigenin (2) & \\
\hline & Apigenin 7 -O-glucuronide (9) & \\
\hline & Galetin 3,6-dimethyl ether (39) & \\
\hline & Luteolin (19) & \\
\hline & Quercetin (44) & \\
\hline & Quercetin 3-O-glucuronide (57) & \\
\hline & Santin (41) & \\
\hline \multirow{6}{*}{ A. lycaonica Boiss. \& Heldr. * } & Apigenin (2) & \multirow{6}{*}[36]{} \\
\hline & 8-Hydroxysalvigenin (6) & \\
\hline & Luteolin (19) & \\
\hline & Naringenin (66) & \\
\hline & Quercetin (44) & \\
\hline & Rutin (58) & \\
\hline \multirow{10}{*}{ A. lycaonica Boiss. \& Heldr. * } & Apigenin (2) & \multirow{9}{*}[27]{} \\
\hline & Fisetin (35) & \\
\hline & Hesperidin (29) & \\
\hline & Hyperoside (59) & \\
\hline & Kaempferol (37) & \\
\hline & Luteolin (19) & \\
\hline & Naringenin (66) & \\
\hline & Quercetin (44) & \\
\hline & Rutin (58) & \\
\hline & Luteolin (isolated) (19) & \multirow{7}{*}[37]{} \\
\hline \multirow{6}{*}{$\begin{array}{l}\text { A. magnifica Hiemerl ex } \\
\text { Hub.-Mor. * }\end{array}$} & Apigenin 6,8-di C-hexoside (vicenin-2 isomer) ${ }^{\#}$ & \\
\hline & Apigenin 6-C-pentoside-8-C-hexosidec $\#$ & \\
\hline & Diosmetin 8-C-glucoside (orientin $4^{\prime}$-methyl ether) & \\
\hline & (28) & \\
\hline & Eupatilin (isolated) (23a) & \\
\hline & Vitexin (10) & \\
\hline & 6-OH-Luteolin-7-O- $\beta$-D-glucoside (30) & \\
\hline A millefolium I & Apigenin $7-O-\beta$-D-glucoside (8) & \\
\hline A. millefolıum L. & Luteolin (19) & [38] \\
\hline & Luteolin 7-O- $\beta$-D-glucoside (24) & \\
\hline
\end{tabular}


Table 2. Cont.

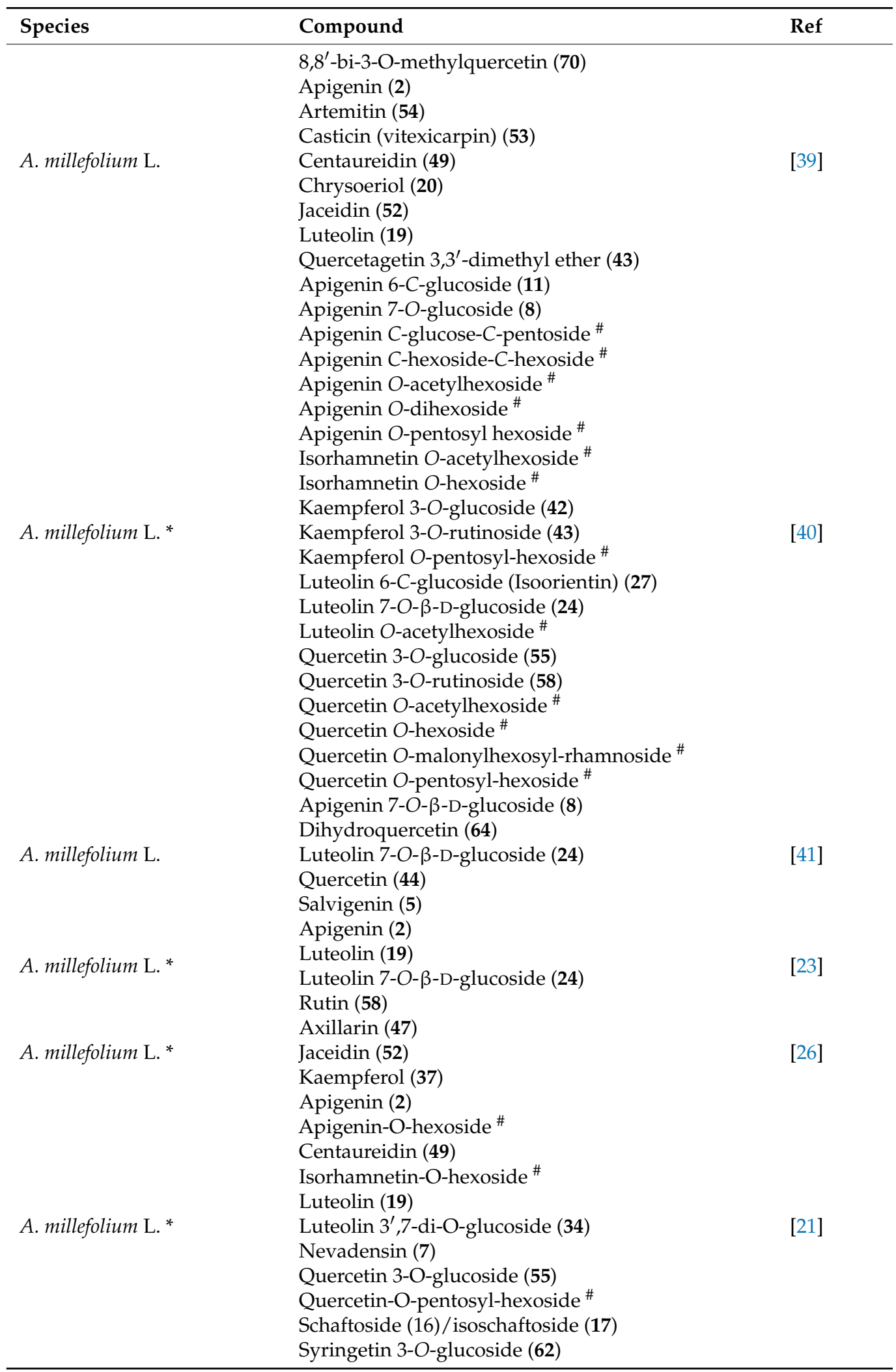


Table 2. Cont.

\begin{tabular}{|c|c|c|}
\hline Species & Compound & Ref \\
\hline A. millefolium L. * & $\begin{array}{l}\text { Apigenin (2) } \\
\text { Apigenin 7-O-glucoside (8) } \\
\text { Apigenin-6,8-di-C-hexoside }{ }^{\#} \mathrm{C} \\
\text { entaureidin (49) } \\
\text { Kaempferol 3-O-rutinoside }{ }^{\#} \\
\text { Kaempferol 3-O-rutinoside }{ }^{\#} \\
\text { Luteolin (19) } \\
\text { Luteolin-hexoside \# } \\
\text { Nevadensin (7) } \\
\text { Quercetin 3-O-rutinoside (58) } \\
\text { Quercetin-O-hexoside Quercetin-O-hexoside }\end{array}$ & [22] \\
\hline $\begin{array}{l}\text { A. monocephala Boiss. \& } \\
\text { Balansa }\end{array}$ & $\begin{array}{l}\text { 8-Hydroxysalvigenin (6) } \\
\text { Luteolin (19) } \\
\text { Naringenin (66) }\end{array}$ & [42] \\
\hline A. moschata W. ${ }^{*}$ & $\begin{array}{l}\text { Apigenin (2) } \\
\text { Apigenin 7-O- } \beta \text {-D-glucoside (8) } \\
\text { Isorhamnetin 3-O-glucosyl } \\
\text { Isorhamnetin 3-O-rutinosyl } \\
\text { Kaempferol 3-O-glucoside (42) } \\
\text { Luteolin (19) } \\
\text { Luteolin 7-O- } \beta \text {-D-glucoside (24) }\end{array}$ & [43] \\
\hline A. moschata W. * & $\begin{array}{l}\text { Apigenin (2) } \\
\text { Apigenin 7-O-glucoside (8) } \\
\text { Isorhamnetin 3-O-glucoside (60) } \\
\text { Isorhamnetin 3-O-rutinoside (61) } \\
\text { Kaempferol 3-O-glucoside (42) } \\
\text { Luteolin (19) } \\
\text { Luteolin 7-O-glucoside (24) }\end{array}$ & {$[44]$} \\
\hline A. moschata W. * & $\begin{array}{l}\text { Apigenin (2) } \\
\text { Apigenin-O-hexoside \# } \\
\text { Centaureidin (49) } \\
\text { Dimethoxy-myricetin-hexoside \# } \\
\text { Isorhamnetin-O-hexoside }{ }^{\#} \\
\text { Luteolin (19) } \\
\text { Mearnsetin-hexoside \# } \\
\text { Nevadensin (7)Syringetin 3-O-glucoside (62) }\end{array}$ & [21] \\
\hline A. nobilis L. * & $\begin{array}{l}\text { Apigenin (2) } \\
\text { Luteolin (19) } \\
\text { Luteolin 7-O- } \beta \text {-D-glucoside (24) } \\
\text { Quercetin (44) } \\
\text { Rutin (58) }\end{array}$ & [23] \\
\hline A. nobilis L. * & $\begin{array}{l}\text { Apigenin-6,8-di-C-glucoside (14) } \\
\text { 5-Demethylsinensetin (23) } \\
\text { Isoorientin (27) } \\
\text { Isoschaftoside (17) } \\
\text { Luteolin (19) } \\
\text { Luteolin 7-O- } \beta \text {-D-glucoside (24) } \\
\text { Orientin (26) } \\
\text { Quercetin (44) } \\
\text { Vitexin (10) }\end{array}$ & [45] \\
\hline A. pachycephala Rech. * & $\begin{array}{l}\text { Apigenin (2) } \\
\text { Luteolin (19) } \\
\text { Luteolin 7-O- } \beta \text {-D-glucoside (24) } \\
\text { Quercetin (44) } \\
\text { Rutin (58) }\end{array}$ & [23] \\
\hline
\end{tabular}


Table 2. Cont.

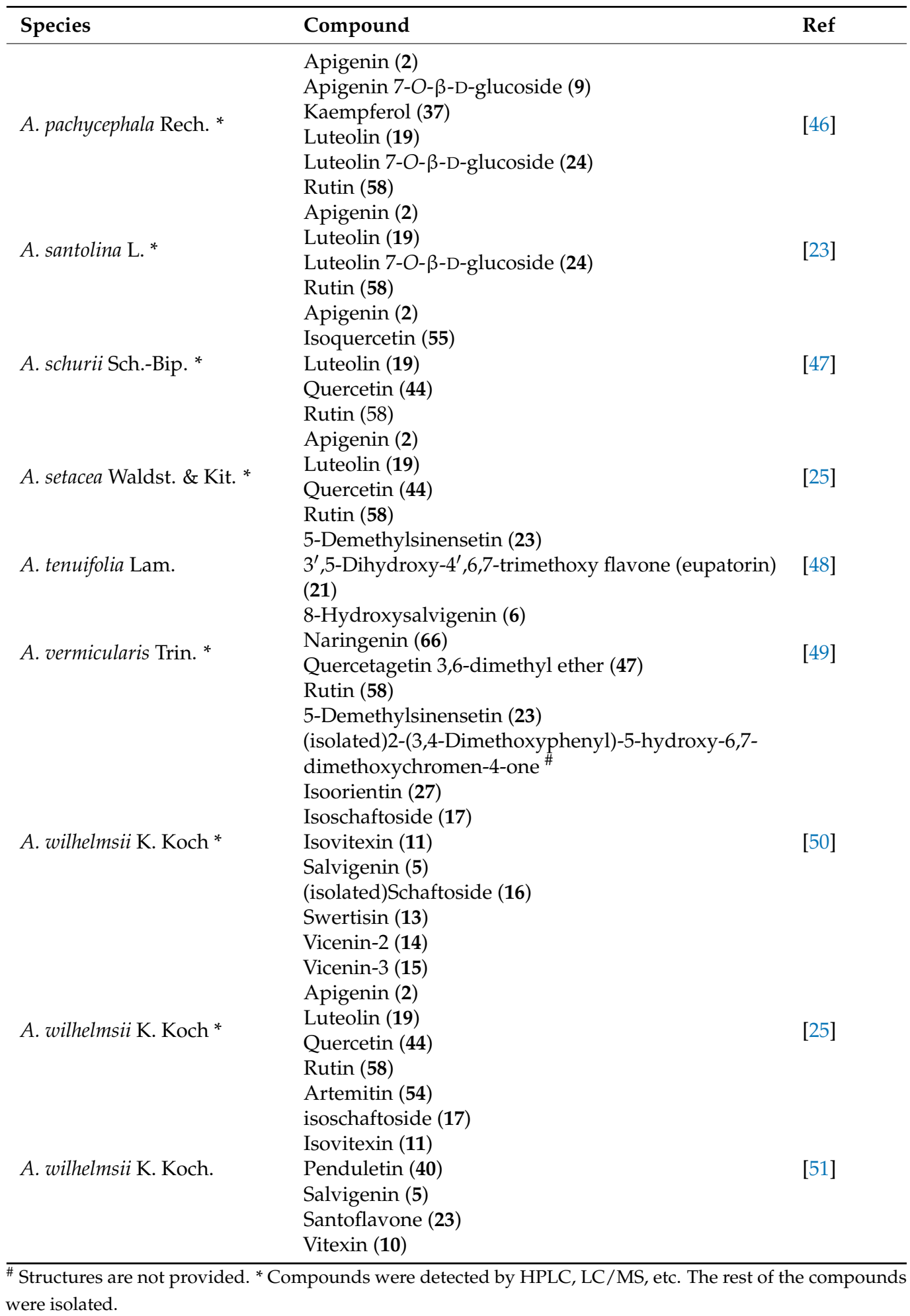


Table 3. Chemical structures of the flavone derivatives from Achillea spp.

\begin{tabular}{|c|c|c|c|c|c|c|}
\hline (c) & $\mathbf{R}_{6}$ & $\mathbf{R}_{7}$ & $\mathbf{R}_{8}$ & $\mathbf{R}_{3}$ & $\mathbf{R}_{\mathbf{4}}$ & $\mathbf{R}_{\mathbf{5}}$ \\
\hline Chrysin (1) & $\mathrm{H}$ & $\mathrm{OH}$ & $\mathrm{H}$ & $\mathrm{H}$ & $\mathrm{H}$ & $\mathrm{H}$ \\
\hline Apigenin (2) & $\mathrm{H}$ & $\mathrm{OH}$ & $\mathrm{H}$ & $\mathrm{H}$ & $\mathrm{OH}$ & $\mathrm{H}$ \\
\hline 7-O-methyl apigenin (genkwanin) (3) & $\mathrm{H}$ & $\mathrm{OCH}_{3}$ & $\mathrm{H}$ & $\mathrm{H}$ & $\mathrm{OH}$ & $\mathrm{H}$ \\
\hline Cirsimaritin (4) & $\mathrm{OCH}_{3}$ & $\mathrm{OCH}_{3}$ & $\mathrm{H}$ & $\mathrm{H}$ & $\mathrm{OH}$ & $\mathrm{H}$ \\
\hline Salvigenin (5) & $\mathrm{OCH}_{3}$ & $\mathrm{OCH}_{3}$ & $\mathrm{H}$ & $\mathrm{H}$ & $\mathrm{OCH}_{3}$ & $\mathrm{H}$ \\
\hline 8-Hydroxysalvigenin (6) & $\mathrm{OCH}_{3}$ & $\mathrm{OCH}_{3}$ & $\mathrm{OH}$ & $\mathrm{H}$ & $\mathrm{OCH}_{3}$ & $\mathrm{H}$ \\
\hline Nevadensin (7) & $\mathrm{OCH}_{3}$ & $\mathrm{OH}$ & $\mathrm{OCH}_{3}$ & $\mathrm{H}$ & $\mathrm{OCH}_{3}$ & $\mathrm{H}$ \\
\hline $\begin{array}{l}\text { Apigenin } 7-O \text {-glucoside } \\
(\operatorname{cosmosiin})(8)\end{array}$ & $\mathrm{H}$ & O-Glc & $\mathrm{H}$ & $\mathrm{H}$ & $\mathrm{OH}$ & $\mathrm{H}$ \\
\hline Apigenin 7-O-glucuronide (9) & $\mathrm{H}$ & O-Gluc & $\mathrm{H}$ & $\mathrm{H}$ & $\mathrm{OH}$ & $\mathrm{H}$ \\
\hline $\begin{array}{l}\text { Apigenin } 8-C \text {-glucoside } \\
\text { (vitexin) (10) }\end{array}$ & $\mathrm{H}$ & $\mathrm{OH}$ & C-Glc & $\mathrm{H}$ & $\mathrm{OH}$ & $\mathrm{H}$ \\
\hline $\begin{array}{c}\text { Apigenin 6-C-glucoside } \\
\text { (isovitexin, saponaretin) (11) }\end{array}$ & C-Glc & $\mathrm{OH}$ & $\mathrm{H}$ & $\mathrm{H}$ & $\mathrm{OH}$ & $\mathrm{H}$ \\
\hline Isovitexin $4^{\prime}$-methyl ether (12) & C-Glc & $\mathrm{OH}$ & $\mathrm{H}$ & $\mathrm{H}$ & $\mathrm{OCH}_{3}$ & $\mathrm{H}$ \\
\hline Swertisin (13) & C-Glc & $\mathrm{OCH}_{3}$ & $\mathrm{H}$ & $\mathrm{H}$ & $\mathrm{OH}$ & $\mathrm{H}$ \\
\hline $\begin{array}{l}\text { Apigenin 6,8-C-diglucoside } \\
\text { (vicenin-2) (14) }\end{array}$ & C-Glc & $\mathrm{OH}$ & C-Glc & $\mathrm{H}$ & $\mathrm{OH}$ & $\mathrm{H}$ \\
\hline $\begin{array}{l}\text { Vicenin-3 (15) } \\
\text { Apigenin }\end{array}$ & C-Glc & $\mathrm{OH}$ & C-Xyl & $\mathrm{H}$ & $\mathrm{OH}$ & $\mathrm{H}$ \\
\hline $\begin{array}{l}\text { 6-C-glucoside- } 8-C \text {-arabinoside } \\
\text { (schaftoside) (16) }\end{array}$ & C-Glc & $\mathrm{OH}$ & C-Ara & $\mathrm{H}$ & $\mathrm{OH}$ & $\mathrm{H}$ \\
\hline $\begin{array}{c}\text { Apigenin } \\
\text { 6-C-arabinoside-8-C-glucoside } \\
\text { (isoschaftoside) }(\mathbf{1 7})\end{array}$ & C-Ara & $\mathrm{OH}$ & C-Glc & $\mathrm{H}$ & $\mathrm{OH}$ & $\mathrm{H}$ \\
\hline $\begin{array}{c}\text { Acacetin } \\
\text { 6-C-(6"-acetyl- } \beta \text {-D-(glucopyranoside)- } \\
\text { 8-C- } \alpha \text {-L-arabinopyranoside } \\
\text { (18) }\end{array}$ & $\begin{array}{l}\text { C-[6"- } \\
\text { acetyl]- } \\
\text { Glc }\end{array}$ & $\mathrm{OH}$ & C-Ara & $\mathrm{H}$ & $\mathrm{OCH}_{3}$ & $\mathrm{H}$ \\
\hline Luteolin (19) & $\mathrm{H}$ & $\mathrm{OH}$ & $\mathrm{H}$ & $\mathrm{OH}$ & $\mathrm{OH}$ & $\mathrm{H}$ \\
\hline Chrysoeriol (20) & $\mathrm{H}$ & $\mathrm{OH}$ & $\mathrm{H}$ & $\mathrm{OCH}_{3}$ & $\mathrm{OH}$ & $\mathrm{H}$ \\
\hline Eupatorin (21) & $\mathrm{OCH}_{3}$ & $\mathrm{OCH}_{3}$ & $\mathrm{H}$ & $\mathrm{OH}$ & $\mathrm{OCH}_{3}$ & $\mathrm{H}$ \\
\hline Cirsiliol (22) & $\mathrm{OCH}_{3}$ & $\mathrm{OCH}_{3}$ & $\mathrm{H}$ & $\mathrm{OH}$ & $\mathrm{OH}$ & $\mathrm{H}$ \\
\hline Eupatilin (23a) & $\mathrm{OCH}_{3}$ & $\mathrm{OH}$ & $\mathrm{H}$ & $\mathrm{OCH}_{3}$ & $\mathrm{OCH}_{3}$ & $\mathrm{H}$ \\
\hline $\begin{array}{c}\text { Eupatilin 7-methyl ether } \\
\text { (5-demethylsinensetin; santoflavone) } \\
\text { (23) }\end{array}$ & $\mathrm{OCH}_{3}$ & $\mathrm{OCH}_{3}$ & $\mathrm{H}$ & $\mathrm{OCH}_{3}$ & $\mathrm{OCH}_{3}$ & $\mathrm{H}$ \\
\hline Luteolin 7-O- $\beta$-D-glucoside (24) & $\mathrm{H}$ & O-Glc & $\mathrm{H}$ & $\mathrm{OH}$ & $\mathrm{OH}$ & $\mathrm{H}$ \\
\hline Luteolin $4^{\prime}-O-\beta$-D-glucoside (25) & $\mathrm{H}$ & $\mathrm{OH}$ & $\mathrm{H}$ & $\mathrm{OH}$ & $\begin{array}{l}\mathrm{O}- \\
\text { Glc }\end{array}$ & $\mathrm{H}$ \\
\hline $\begin{array}{l}\text { Luteolin } 8 \text {-C-glucoside } \\
\text { (orientin) (26) }\end{array}$ & $\mathrm{H}$ & $\mathrm{OH}$ & C-Glc & $\mathrm{OH}$ & $\mathrm{OH}$ & $\mathrm{H}$ \\
\hline $\begin{array}{l}\text { Luteolin 6-C-glucoside } \\
\quad \text { (isoorientin) (27) }\end{array}$ & C-Glc & $\mathrm{OH}$ & $\mathrm{H}$ & $\mathrm{OH}$ & $\mathrm{OH}$ & $\mathrm{H}$ \\
\hline Orientin $4^{\prime}$-methyl ether (28) & C-Glc & $\mathrm{OH}$ & $\mathrm{H}$ & $\mathrm{OH}$ & $\mathrm{OCH}_{3}$ & $\mathrm{H}$ \\
\hline $\begin{array}{l}\text { Acacetin 7-O-rutinoside (hesperidin) } \\
\text { (29) }\end{array}$ & $\mathrm{H}$ & O-Rut & $\mathrm{H}$ & $\mathrm{OH}$ & $\mathrm{OCH}_{3}$ & $\mathrm{H}$ \\
\hline 6-OH-luteolin 7-O- $\beta$-D-glucoside (30) & $\mathrm{OH}$ & O-Glc & $\mathrm{H}$ & $\mathrm{OH}$ & $\mathrm{OH}$ & $\mathrm{H}$ \\
\hline Chrysoeriol 7-O- $\beta$-D-glucoside (31) & $\mathrm{OH}$ & O-Glc & $\mathrm{H}$ & $\mathrm{OCH}_{3}$ & $\mathrm{OH}$ & $\mathrm{H}$ \\
\hline Chrysoeriol 7-O-rutinoside (32) & $\mathrm{OH}$ & O-Rut & $\mathrm{H}$ & $\mathrm{OCH}_{3}$ & $\mathrm{OH}$ & $\mathrm{H}$ \\
\hline Tricin 7-O- $\beta$-D-glucoside (33) & $\mathrm{H}$ & O-Gluc & $\mathrm{H}$ & $\mathrm{OCH}_{3}$ & $\mathrm{OH}$ & $\mathrm{OCH}_{3}$ \\
\hline Luteolin $3^{\prime}, 7$-di-O-glucoside (34) & $\mathrm{H}$ & O-Glc & $\mathrm{H}$ & $\begin{array}{l}\text { O- } \\
\text { Glc }\end{array}$ & $\mathrm{OH}$ & $\mathrm{H}$ \\
\hline
\end{tabular}


Table 4. Chemical structures of the 3-Hydroxyflavone (flavonol) derivatives from Achillea spp.

\begin{tabular}{|c|c|c|c|c|c|c|c|c|}
\hline$\underbrace{\mathrm{R}_{8}}_{1}$ & $\mathbf{R}_{3}$ & $\mathbf{R}_{5}$ & $\mathbf{R}_{6}$ & $\mathbf{R}_{7}$ & $\mathbf{R}_{8}$ & $\mathbf{R}_{\mathbf{3}^{\prime}}$ & $\mathbf{R}_{4^{\prime}}$ & $\mathbf{R}_{5^{\prime}}$ \\
\hline Fisetin (35) & $\mathrm{OH}$ & $\mathrm{H}$ & $\mathrm{H}$ & $\mathrm{OH}$ & $\mathrm{H}$ & $\mathrm{H}$ & $\mathrm{OH}$ & $\mathrm{OH}$ \\
\hline Galangin (36) & $\mathrm{OH}$ & $\mathrm{OH}$ & $\mathrm{H}$ & $\mathrm{OH}$ & $\mathrm{H}$ & $\mathrm{H}$ & $\mathrm{H}$ & $\mathrm{H}$ \\
\hline Kaempferol (37) & $\mathrm{OH}$ & $\mathrm{OH}$ & $\mathrm{H}$ & $\mathrm{OH}$ & $\mathrm{H}$ & $\mathrm{H}$ & $\mathrm{OH}$ & $\mathrm{H}$ \\
\hline 3,8-Dimethylherbacetin (38) & $\mathrm{OCH}_{3}$ & $\mathrm{OH}$ & $\mathrm{H}$ & $\mathrm{OH}$ & $\mathrm{OCH}_{3}$ & $\mathrm{H}$ & $\mathrm{OH}$ & $\mathrm{H}$ \\
\hline $\begin{array}{c}\text { Galetin 3,6-dimethyl ether } \\
\left(5,7,4^{\prime} \text {-trihydroxy-3,6-dimethoxyflavanone) (39) }\right.\end{array}$ & $\mathrm{OCH}_{3}$ & $\mathrm{OH}$ & $\mathrm{OCH}_{3}$ & $\mathrm{OH}$ & $\mathrm{H}$ & $\mathrm{H}$ & $\mathrm{OH}$ & $\mathrm{H}$ \\
\hline Penduletin (40) & $\mathrm{OCH}_{3}$ & $\mathrm{OH}$ & $\mathrm{OCH}_{3}$ & $\mathrm{OCH}_{3}$ & $\mathrm{H}$ & $\mathrm{H}$ & $\mathrm{OH}$ & $\mathrm{H}$ \\
\hline Santin (41) & $\mathrm{OCH}_{3}$ & $\mathrm{OH}$ & $\mathrm{OCH}_{3}$ & $\mathrm{OH}$ & $\mathrm{H}$ & $\mathrm{H}$ & $\mathrm{OCH}_{3}$ & $\mathrm{H}$ \\
\hline Kaempferol 3-O-glucoside (42) & O-Glc & $\mathrm{OH}$ & $\mathrm{H}$ & $\mathrm{OH}$ & $\mathrm{H}$ & $\mathrm{H}$ & $\mathrm{OH}$ & $\mathrm{H}$ \\
\hline Kaempferol 3-O-rutinoside (43) & O-Rut & $\mathrm{OH}$ & $\mathrm{H}$ & $\mathrm{OH}$ & $\mathrm{H}$ & $\mathrm{H}$ & $\mathrm{OH}$ & $\mathrm{H}$ \\
\hline Quercetin (44) & $\mathrm{OH}$ & $\mathrm{OH}$ & $\mathrm{H}$ & $\mathrm{OH}$ & $\mathrm{H}$ & $\mathrm{OH}$ & $\mathrm{OH}$ & $\mathrm{H}$ \\
\hline Isorhamnetin (45) & $\mathrm{OH}$ & $\mathrm{OH}$ & $\mathrm{H}$ & $\mathrm{OH}$ & $\mathrm{H}$ & $\mathrm{OCH}_{3}$ & $\mathrm{OH}$ & $\mathrm{H}$ \\
\hline 5,7-dihydroxy-3,3', $4^{\prime}$-trimethoxy flavone (46) & $\mathrm{OCH}_{3}$ & $\mathrm{OH}$ & $\mathrm{H}$ & $\mathrm{OH}$ & $\mathrm{H}$ & $\mathrm{OCH}_{3}$ & $\mathrm{OCH}_{3}$ & $\mathrm{H}$ \\
\hline Quercetagetin 3,6-dimethyl ether (Axillarin) (47) & $\mathrm{OCH}_{3}$ & $\mathrm{OH}$ & $\mathrm{OCH}_{3}$ & $\mathrm{OH}$ & $\mathrm{H}$ & $\mathrm{OH}$ & $\mathrm{OH}$ & $\mathrm{H}$ \\
\hline Quercetagetin 3,3'-dimethyl ether (48) & $\mathrm{OCH}_{3}$ & $\mathrm{OH}$ & $\mathrm{OH}$ & $\mathrm{OH}$ & $\mathrm{H}$ & $\mathrm{OCH}_{3}$ & $\mathrm{OH}$ & $\mathrm{H}$ \\
\hline Centaureidin (49) & $\mathrm{OCH}_{3}$ & $\mathrm{OH}$ & $\mathrm{OCH}_{3}$ & $\mathrm{OH}$ & $\mathrm{H}$ & $\mathrm{OH}$ & $\mathrm{OCH}_{3}$ & $\mathrm{H}$ \\
\hline Chrysosplenol B (50) & $\mathrm{OCH}_{3}$ & $\mathrm{OH}$ & $\mathrm{OCH}_{3}$ & $\mathrm{OCH}_{3}$ & $\mathrm{H}$ & $\mathrm{OH}$ & $\mathrm{OCH}_{3}$ & $\mathrm{H}$ \\
\hline Chrysosplenetin (50a) & $\mathrm{OCH}_{3}$ & $\mathrm{OH}$ & $\mathrm{OCH}_{3}$ & $\mathrm{OCH}_{3}$ & $\mathrm{H}$ & $\mathrm{OCH}_{3}$ & $\mathrm{OH}$ & $\mathrm{H}$ \\
\hline Quercetin 3,6,7-trimethyl ether (Chrysosplenol D) & $\mathrm{OCH}_{3}$ & $\mathrm{OH}$ & $\mathrm{OCH}_{3}$ & $\mathrm{OCH}_{3}$ & $\mathrm{H}$ & $\mathrm{OH}$ & $\mathrm{OH}$ & $\mathrm{H}$ \\
\hline Jaceidin (Quercetagetin 3,6,3'-trimethyl ether) (52) & $\mathrm{OCH}_{3}$ & $\mathrm{OH}$ & $\mathrm{OCH}_{3}$ & $\mathrm{OH}$ & $\mathrm{H}$ & $\mathrm{OCH}_{3}$ & $\mathrm{OH}$ & $\mathrm{H}$ \\
\hline Casticin (53) & $\mathrm{OCH}_{3}$ & $\mathrm{OH}$ & $\mathrm{OCH}_{3}$ & $\mathrm{OCH}_{3}$ & $\mathrm{H}$ & $\mathrm{OH}$ & $\mathrm{OCH}_{3}$ & $\mathrm{H}$ \\
\hline Artemitin (54) & $\mathrm{OCH}_{3}$ & $\mathrm{OH}$ & $\mathrm{OCH}_{3}$ & $\mathrm{OCH}_{3}$ & $\mathrm{H}$ & $\mathrm{OCH}_{3}$ & $\mathrm{OCH}_{3}$ & $\mathrm{H}$ \\
\hline $\begin{array}{l}\text { Quercetin 3-O-glucoside } \\
\text { (isoquercetin) (55) }\end{array}$ & O-Glc & $\mathrm{OH}$ & $\mathrm{H}$ & $\mathrm{OH}$ & $\mathrm{H}$ & $\mathrm{OH}$ & $\mathrm{OH}$ & $\mathrm{H}$ \\
\hline Quercitin (56) & O-Rha & $\mathrm{OH}$ & $\mathrm{H}$ & $\mathrm{OH}$ & $\mathrm{H}$ & $\mathrm{OH}$ & $\mathrm{OH}$ & $\mathrm{H}$ \\
\hline Quercetin 3-O-glucuronide (57) & O-Glc & $\mathrm{OH}$ & $\mathrm{H}$ & $\mathrm{OH}$ & $\mathrm{H}$ & $\mathrm{OH}$ & $\mathrm{OH}$ & $\mathrm{H}$ \\
\hline $\begin{array}{l}\text { Rutin } \\
\text { (quercetin 3-O-rutinoside) (58) }\end{array}$ & O-Rut & $\mathrm{OH}$ & $\mathrm{H}$ & $\mathrm{OH}$ & $\mathrm{H}$ & $\mathrm{OH}$ & $\mathrm{OH}$ & $\mathrm{H}$ \\
\hline Hyperoside (quercetin 3-O-galactoside) (59) & O-Gal & $\mathrm{OH}$ & $\mathrm{H}$ & $\mathrm{OH}$ & $\mathrm{H}$ & $\mathrm{OH}$ & $\mathrm{OH}$ & $\mathrm{H}$ \\
\hline Isorhamnetin 3-O-glucoside (60) & O-Glc & $\mathrm{OH}$ & $\mathrm{H}$ & $\mathrm{OH}$ & $\mathrm{H}$ & $\mathrm{OCH}_{3}$ & $\mathrm{OH}$ & $\mathrm{H}$ \\
\hline Isorhamnetin 3-O-rutinoside (61) & O-Rut & $\mathrm{OH}$ & $\mathrm{H}$ & $\mathrm{OH}$ & $\mathrm{H}$ & $\mathrm{OCH}_{3}$ & $\mathrm{OH}$ & $\mathrm{H}$ \\
\hline Syringetin 3-O-glucoside (62) & O-Glc & $\mathrm{OH}$ & $\mathrm{H}$ & $\mathrm{OH}$ & $\mathrm{H}$ & $\mathrm{OCH}_{3}$ & $\mathrm{OH}$ & $\mathrm{OCH}_{3}$ \\
\hline $\begin{array}{c}\text { Quercetin 3-O-arabinosyl }(1 \rightarrow 6) \text { glucoside (Quercetin } \\
\text { 3-O-vicianoside) (63) }\end{array}$ & $\begin{array}{c}\text { O-Ara } \\
1 \rightarrow 6 \\
\text { Glc }\end{array}$ & $\mathrm{OH}$ & $\mathrm{H}$ & $\mathrm{OH}$ & $\mathrm{H}$ & $\mathrm{OH}$ & $\mathrm{OH}$ & $\mathrm{H}$ \\
\hline
\end{tabular}


Table 4. Cont.

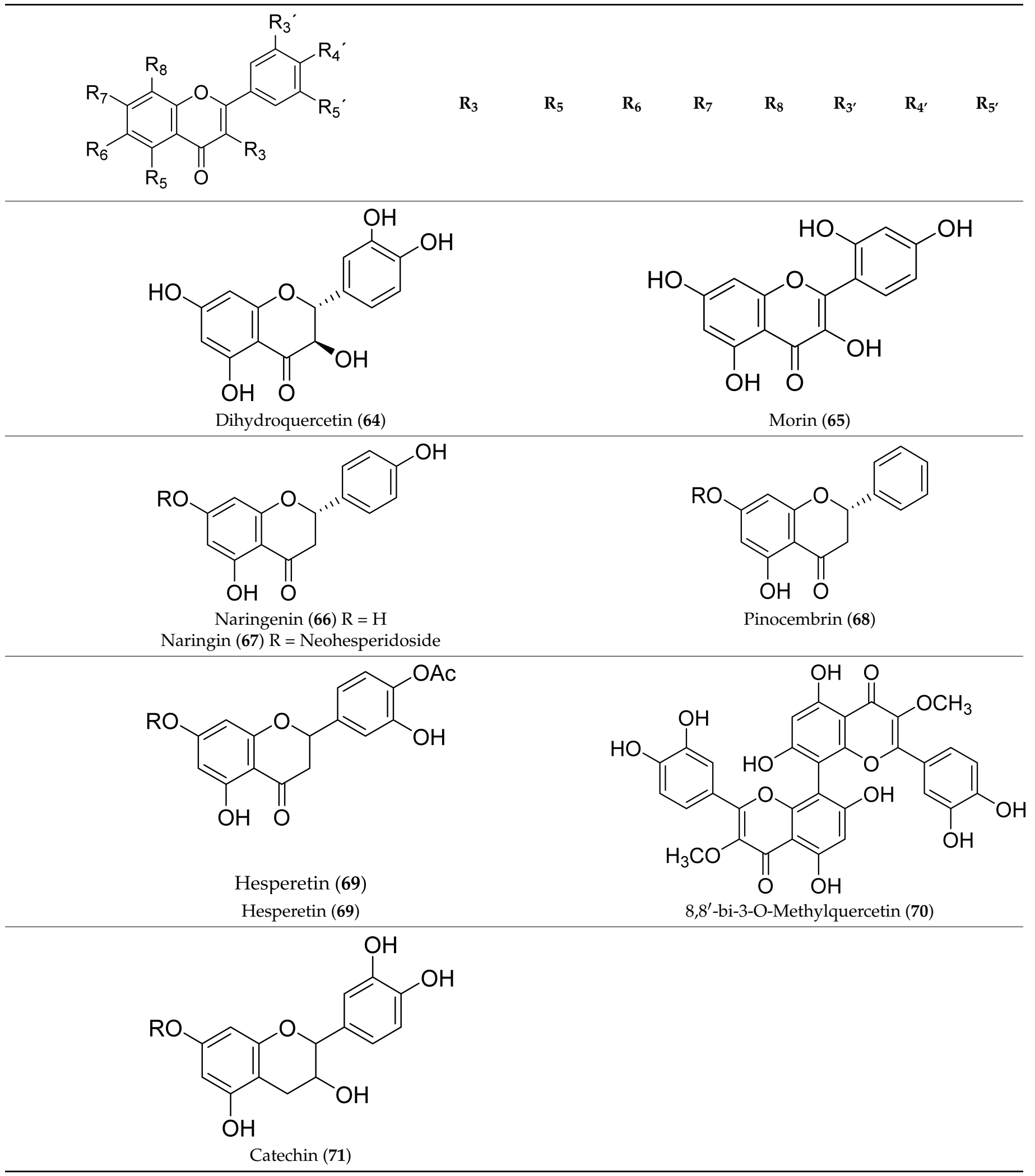

Glc: glucoside; Gluc: glucuronide; Rut: rutinoside; Ara: arabinoside; Rha: rhamnoside. 


\subsubsection{Phenolic and Quinic Acids Derivatives}

Regarding the phenolic profile of Achillea spp. reported in the updated literature (2011-2021), the genus is characterised by the occurrence of benzoic, caffeic, chlorogenic and caffeoylquinic acid derivatives (Tables 5 and 6).

Chlorogenic acid (81) is one of the most abundant compounds found in A. alpina, A. asiatica, A. biebersteinii, A. coarctata, A. distans, A. kotschyi, A. ligustica, A. lycaonica, A. millefolium, A. multifidi, A. schurii, A. setacea, A. vermicularis and A. wilhelmsii (Table 5). Moreover, isomers of the aforementioned compound were also reported from A. alpina [56], A. biebersteinii [26], A. cretica [28], A. millefolium and A. moschata [42]. Caffeic acid (94) is also abundant, as it was found in 17 Achillea spp. (A. biebersteinii, A. coarctata, A. distans, A. kotschyi Boiss. subsp. kotschyi, A. ligustica, A. lycaonica, A. millefolium, A. schurii, A. setacea, A. vermicularis, A. wilhelmsii, etc.) (Table 5). Though, ferulic acid (95) was reported only from A. wilhelmsii [50], while coumaric acid (93) was identified in A. schurii [47] and A. kotschyi Boiss. subsp. kotschyi [27]. Concerning the quinic acid (80) derivatives related to this genus, such compounds encompass both caffeoyl- or coumaroyl-esters. The 5-coumaroyl quinic acid (89) and its methyl ester (92) were reported from A. alpina [19], while other coumaroyl quinic isomers were identified in A. biebersteinii and in A. millefolium [26]. Furthermore, many studies revealed the high presence on dicaffeoylquinic acid derivatives, with the 3,4-O-/4,5-O-dicaffeoyl-quinic acid isomers being the most common [57]. It is noteworthy that only one study unveiled the appearance of the methyl ester of 3,5-dicaffeoylquinic acid (91) in A. alpina [18].

A few benzoic acids were found in the Achillea genus. For instance, salicylic acid (97a) and benzoic acid (72) were detected by Agar et al. [27] through LC/MS-MS analysis in A. coarctata, A. kotschyi and A. lycaonica. Furthermore, Benedec et al. [47] mentioned the presence of genistic acid (78) in A. schurii [47]. Moreover, the 4-hydroxybenzoic acid (73) was detected only in $A$. coarctata [27]. In the same study, protocatechuic acid (75) was found only in two species, A. kotschyi and A. lycaonica [27]. It is interesting to point out that veratric acid (74) was isolated once from $A$. fragrantissima, and 2,4-dihydroxy-methyl benzoate (79) was isolated only from A. tenuifolia [48].

In addition to all the above-mentioned aglycon derivatives, two glucosyl derivatives namely 1-[4-( $\beta$-D-glucopyranosyloxy)-3,5-dimethoxyphenyl]-propanone (96) and 4-(4'-O$\beta$-D-glucopyranosyl-3', $5^{\prime}$ dimethoxyphenyl)-2-butanone (97) were reported for the first time in the genus Achillea by Zhang et al. [19] from A. alpina. Intriguingly, only one study on $A$. millefolium reported caffeic acid combined with a hexose moiety.

Table 5. Phenolic and quinic acids from Achillea spp.

\begin{tabular}{|c|c|c|}
\hline Species & Compound & Ref \\
\hline \multirow{4}{*}{ A. alpina $\mathrm{L}$. } & Isochlorogenic acid A (86) & \multirow{2}{*}[57]{} \\
\hline & Isochlorogenic acid B (87) & \\
\hline & 5-Coumaroyl quinic acid (89) & \multirow{7}{*}{ [18] } \\
\hline & 5-O-Coumaroyl quinic acid methyl ester (92) & \\
\hline \multirow{5}{*}{ A. alpina $\mathrm{L}$. } & Chlorogenic acid (81) & \\
\hline & Chlorogenic acid methyl ester (90) & \\
\hline & 3,5-Dicaffeoylquinic acid (86) & \\
\hline & Methyl 3,5-dicaffeoyl quinic acid (91) & \\
\hline & $\begin{array}{l}\text { 1-[4-( } \beta \text {-D-Glucopyranosyloxy)-3,5- } \\
\text { dimethoxyphenyl]-propanone }(\mathbf{9 6})\end{array}$ & \\
\hline A. alpina L. & $\begin{array}{l}4-\left(4^{\prime}-O-\beta \text {-D-Glucopyranosyl- }\right. \\
3^{\prime}, 5^{\prime} \text { dimethoxyphenyl)-2-butanone } \\
(\mathbf{9 7})\end{array}$ & {$[19]$} \\
\hline \multirow[t]{2}{*}{ A. asiatica Serg. } & Chlorogenic acid (81) & \multirow[t]{2}{*}[20]{} \\
\hline & p-Coumaric acid derivative ${ }^{\#}$ & \\
\hline \multirow[t]{2}{*}{ A. atrata L. * } & Chlorogenic acid (81) & \multirow[t]{2}{*}{ [21] } \\
\hline & Dicaffeoylquinic acid ${ }^{\#}$ & \\
\hline
\end{tabular}


Table 5. Cont.

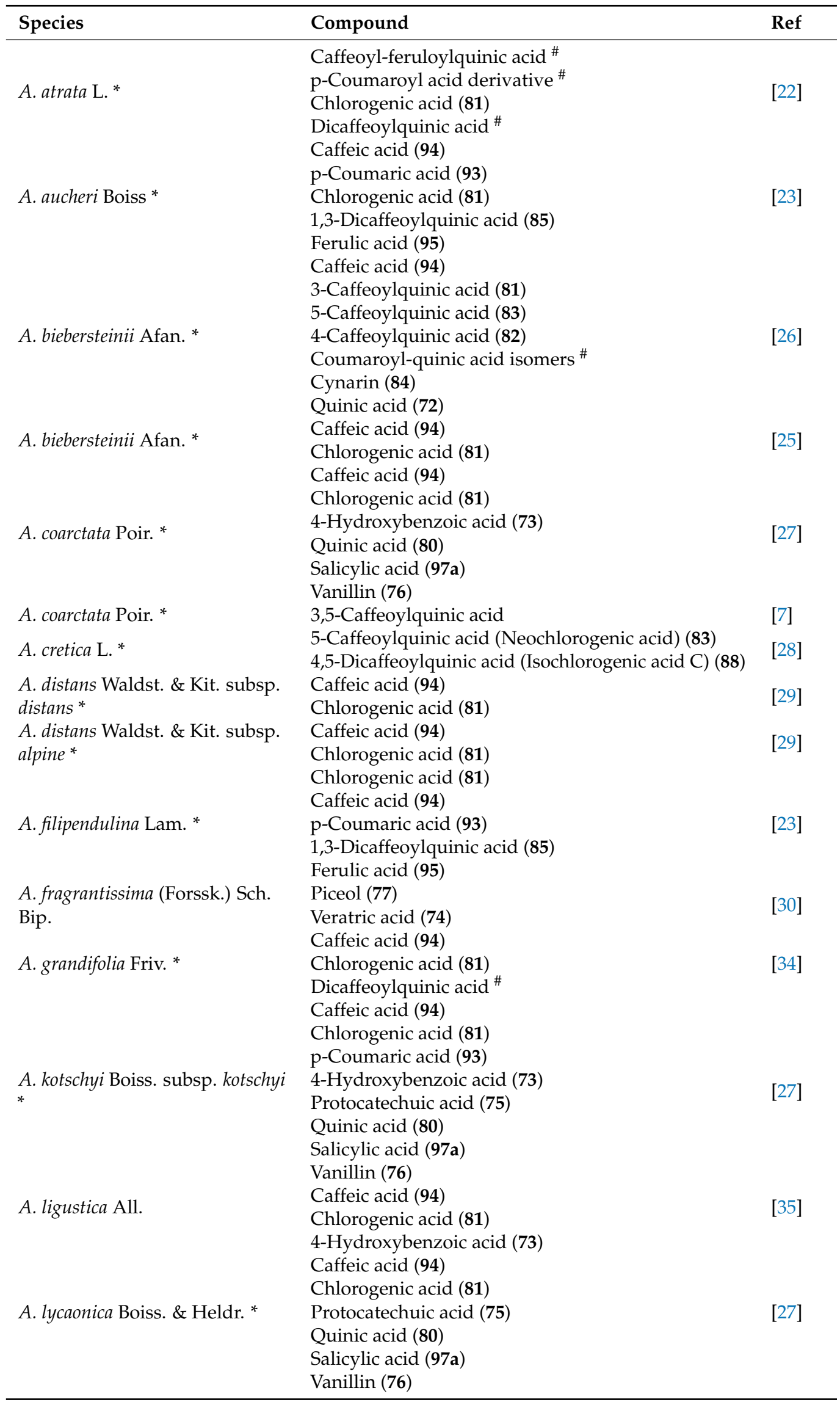


Table 5. Cont.

\begin{tabular}{|c|c|c|}
\hline Species & Compound & Ref \\
\hline \multirow[t]{5}{*}{ A. millefolium $\mathrm{L}$. } & Chlorogenic acid (81) & {$[41]$} \\
\hline & Caffeic acid (94) & \\
\hline & Caffeic acid hexoside $\#$ & \\
\hline & 5-Caffeoylquinic acid (83) & \\
\hline & 3-Caffeoylquinic acid (81) & \\
\hline \multirow[t]{7}{*}{ A. millefolium L. * } & 4-Caffeoylquinic acid (82) & {$[40]$} \\
\hline & 3,4-Dicaffeoylquinic acid (87) & \\
\hline & cis 3,5-Dicaffeoylquinic acid ${ }^{\#}$ & \\
\hline & trans 3,5-Dicaffeoylquinic acid ${ }^{\#}$ & \\
\hline & 4,5-Dicaffeoylquinic acid (88) & \\
\hline & Quinic acid (80) & \\
\hline & 3-Caffeoylquinic acid (81) & \\
\hline \multirow{6}{*}{ A. millefolium L. * } & 5-Caffeoylquinic acid (83) & \\
\hline & 4-Caffeoylquinic acid (82) & {$[26]$} \\
\hline & Coumaroyl-quinic acid isomers \# & \\
\hline & Cynarin $(84)$ & \\
\hline & Caffeic acid (94) & \\
\hline & Chlorogenic acid (81) & \\
\hline \multirow[t]{4}{*}{ A. millefolium L. * } & p-Coumaric acid (93) & {$[23]$} \\
\hline & 1,3-Dicaffeoylquinic acid (85) & \\
\hline & Ferulic acid (95) & \\
\hline & Chlorogenic acid (81) & \\
\hline \multirow{3}{*}{ A. millefolium L. * } & p-Coumaric acid derivative & {$[21]$} \\
\hline & Dicaffeoylquinic acid $\#$ & \\
\hline & Chlorogenic acid (81) & \\
\hline \multirow[t]{2}{*}{ A. millefolium L. * } & p-Coumaroyl acid derivative ${ }^{\#}$ & {$[22]$} \\
\hline & Dicaffeoylquinic acid ${ }^{\#}$ & \\
\hline \multirow{3}{*}{ A. moschata W. * } & 5-Caffeoylquinic acid (83) & {$[43]$} \\
\hline & 4,5-Dicaffeoylquinic acid (88) & {$[43]$} \\
\hline & Chlorogenic acid (81) & \\
\hline \multirow[t]{3}{*}{ A. moschata W. * } & p-Coumaric acid derivative & {$[21]$} \\
\hline & Dicaffeoylquinic acid ${ }^{\#}$ & \\
\hline & 5-Caffeoylquinic acid (83) & \\
\hline \multirow[t]{2}{*}{ A. moschata W. * } & 4,5-Dicaffeoylquinic acid (88) & {$[44]$} \\
\hline & Disuccinylcaffeoylquinic acid ${ }^{\#}$ & \\
\hline \multirow{4}{*}{ A. multifidi (D.C.) Boiss. * } & Chlorogenic acid (81) & \\
\hline & Dicaffeoylquinic acid ${ }^{\#}$ & [58] \\
\hline & Caffeic acid (94) & \\
\hline & Chlorogenic acid (81) & \\
\hline \multirow[t]{3}{*}{ A. nobilis L. * } & p-Coumaric acid (93) & {$[23]$} \\
\hline & 1,3-Dicaffeoylquinic acid (85) & \\
\hline & Ferulic acid (95) & \\
\hline \multirow[t]{2}{*}{ A. nobilis L. * } & Chlorogenic acid (81) & {$[45]$} \\
\hline & Caffeic acid (94) & \\
\hline \multirow[t]{4}{*}{ A. pachycephala Rech. * } & Chlorogenic acid (81) & [46] \\
\hline & 1,3-Dicaffeoylquinic acid (85) & \\
\hline & Caffeic acid (94) & \\
\hline & Chlorogenic acid (81) & \\
\hline \multirow[t]{4}{*}{ A. santolina $\mathrm{L} .{ }^{*}$} & p-Coumaric acid (93) & {$[23]$} \\
\hline & 1,3-Dicaffeoylquinic acid (85) & \\
\hline & Ferulic acid (95) & \\
\hline & Caffeic acid (94) & \\
\hline \multirow{3}{*}{ A. schurii Sch.-Bip. * } & Genistic acid (78) & \\
\hline & Chlorogenic acid (81) & {$[47]$} \\
\hline & p-Coumaric acid (93) & \\
\hline \multirow{2}{*}{ A. setacea Waldst. \& Kit. * } & Caffeic acid (94) & {$[25]$} \\
\hline & Chlorogenic acid (73) & [25] \\
\hline
\end{tabular}


Table 5. Cont.

\begin{tabular}{lll}
\hline Species & Compound & Ref \\
\hline A. tenuifolia Lam. & 2,4-Dihydroxy-methyl benzoate (79) & Caffeic acid (94) \\
A. vermicularis Trin. & Chlorogenic acid (81) & [49] \\
& Dicaffeoylquinic acid \# & \\
A. wilhelmsii K. Koch. * & Caffeic acid (94) & [50] \\
A. wilhelmsii K. Koch * & Ferulic acid (95) & Caffeic acid (94) \\
A. wilhelmsii K. Koch. & Chlorogenic acid (81) & Chlorogenic acid (81) \\
\hline
\end{tabular}

\# Structures are not provided. ${ }^{*}$ Compounds were detected by HPLC, LC/MS, etc. The rest of the compounds were isolated.

\subsubsection{Sesquiterpenes Lactones (SLs)}

The Asteraceae family is characterized by an extraordinary range of structurally modified and highly substituted skeletons of SLs. Due to this structural diversity and their diverse array of biological activities, SLs from Achillea spp. are considered as one of the most interesting phytochemical groups among others of the genus. Although different types of SLs were reported, germacranolides, guaianolides and seco-guaianolides are the most representative classes. Previous studies highlighted the structural features of SLs in Achillea spp. In a review conducted by Si et al. [11], more than 100 compounds were described with guaianolides and germacranolides being the major constituents, by far. The study also reported other skeletal types, such as seco-guaianolides, 12,6 $\alpha$-eudesmanolides and other eudesmanolides. The literature data from the past decade include approximately 35 SLs divided mainly into three sub-groups according to their chemical natures, i.e., guaianolides, seco-guaianolides, germacranolides, which are discussed below (Tables 7-13).

Achillin (103), a costus-type guaianolide, was isolated from A. alpina from Korea [18]. Moreover, achillin (103) and leucodin (102) were isolated from A. millefolium from Mexico [67]. Similarly, leucodin (102) and 1 $\beta, 10 \beta$-epoxy-desacetoxymatricarin (107) were reported for the aerial parts of $A$. wilhelmsii from Syria [50].

Two germacranolides (micranthin and sintenin) and one rarely occurring 8,12-guaianolide $(4 \beta, 10 \alpha$-dihydroxy- $5 \beta, 7 \beta, 8 \beta \mathrm{H}$-guaia-1,11(13)dien-12,8 $\alpha$-olide; 116) were isolated from the ethyl acetate extract of the aerial parts of $A$. biebersteinii, collected from Saudi Arabia [24].

The aerial parts of $A$. clavennae L. (collected in Montenegro) yielded six SLs; three germacranolides, sintenin (136) and two other highly oxygenated, namely $3 \beta, 9 \beta$-diacetoxy-1 $\beta$ hydroperoxy-6 $\beta, 7 \alpha$ H-germacra-4,10(14),11(13)-trien-12,6 $\alpha$-olide (137) and 3,9-diacetoxy-1hydroxy-6 $\beta, 7 \alpha, 11 \mathrm{H}$-germacra-4,10(14)-dien-12,6 $\alpha$-olide, (138), $9 \alpha$-acetoxycanin (102) and two iso-seco-guaianolide, i.e., apressin (106) and $3 \beta$-methoxy-iso-seco-tanapartholide (124) [60].

Papakosta et al. [7] investigated the non-polar extract of the aerial parts of $A$. coarctata from Greece, isolating three common guaianolides; rupicolins A (98) and B (99) and 11,13dihydrodesacetylmatricarin (93), two eudesmanolides; artecalin (140) and 3-acetylridentin (141), and also the seco-guiainolide arteludovicinolide A (122). Furthermore, phytochemical studies in $A$. falcata, an indigenous plant to the Middle Eastern region, indicated the presence of $3 \beta$-methoxy-iso-seco-tanapartholide (124) $[62,63]$. In the study carried out by Tohme et al. [63], six more sesquiterpene lactones were isolated following an acid-base extraction of $A$. falcata aerial parts. Specifically, three secotanapartholides, namely iso-secotanapartholide (123), tanaphillin (128) and 8-hydroxy-3-methoxy-iso-secotanapartholide (132), as well as three highly oxygenated guaianolides, rupin A (110), chrysartemin B (109) and $1 \beta, 2 \beta$-epoxy-3 $\beta, 4 \alpha, 10 \alpha$-trihydroxyguaian-6 $\alpha, 12$-olide (105) were found. 
Table 6. Chemical structures of the phenolic and quinic acid derivatives from Achillea spp.<smiles>[R2]c1cc(C(=O)O)cc([R])c1[R]</smiles>

Vanillin (76)<smiles>CC(=O)c1ccc(O)cc1</smiles>

Piceol (77)<smiles>O=C(O)c1cc(O)ccc1O</smiles>

Genistic acid (78)<smiles>COC(=O)c1ccc(O)cc1O</smiles>

2,4-Dihydroxy-methyl benzoate

Quinic acid $\mathrm{R}_{1}=\mathrm{R}_{2}=\mathrm{R}_{3}=\mathrm{R}_{4}=\mathrm{H}$ (80)

3-Caffeoylquinic acid (chlorogenic acid) $R_{1}=H, R_{2}=$ caffeoyl-, $R_{3}=$ $\mathrm{R}_{4}=\mathrm{H}(\mathbf{8 1})$

4-Caffeoylquinic acid (cryptochlorogenic acid) $\mathrm{R}_{1}=\mathrm{R}_{2}=\mathrm{H}, \mathrm{R}_{3}=$ caffeoyl-, $\mathrm{R}_{4}=\mathrm{H}(\mathbf{8 2})$<smiles>[R20]OC1CC(O[R10])(C(=O)O)CC([R20])C1[R20]</smiles>

5-Caffeoylquinic acid (neohlorogenic acid) $R_{1}=R_{2}=R_{3}=H, R_{4}=$ caffeoyl- (83)

Cynarin (1,5-Dicaffeoylquinic acid) $\mathrm{R}_{1}=\mathrm{R}_{4}=$ caffeoyl-, $\mathrm{R}_{3}=\mathrm{R}_{2}=\mathrm{H}$ (84)

1,3-Dicaffeoylquinic acid $\mathrm{R}_{1}=\mathrm{R}_{2}=$ caffeoyl-, $\mathrm{R}_{3}=\mathrm{R}_{4}=\mathrm{H}$ (85)

Isochlorogenic acid A (3,5-dicaffeoylquinic acid) $R_{1}=R_{3}=H, R_{2}=$ $\mathrm{R}_{4}=$ caffeoyl $-(86)$

Isochlorogenic acid $B\left(3,4\right.$-dicaffeoylquinic acid) $R_{1}=R_{4}=H, R_{2}=$ $\mathrm{R}_{3}=$ caffeoyl-(87)

4,5-Dicaffeoylquinic acid (isochlorogenic acid C) $R_{1}=R_{2}=H, R_{3}=$ $\mathrm{R}_{4}$ = caffeoyl- (88)

5-Coumaroyl quinic acid $\mathrm{R}_{1}=\mathrm{R}_{2}=\mathrm{R}_{3}=\mathrm{HR}_{4}=$ coumaroyl- (89)<smiles>[R20]OC1CC(O[R20])(C(=O)OC)CC([R20])C1O[R]</smiles>

Chlorogenic acid methyl ester $\mathrm{R}_{1}=\mathrm{H}, \mathrm{R}_{2}=$ caffeoyl-, $\mathrm{R}_{3}=\mathrm{R}_{4}=\mathrm{H}$

Methyl 3,5-dicaffeoyl quinic acid $\mathrm{R}_{1}=\mathrm{R}_{3}=\mathrm{H}, \mathrm{R}_{2}=\mathrm{R}_{4}=$ caffeoyl(91)

5-O-Coumaroyl quinic acid methyl ester $\mathrm{R}_{1}=\mathrm{R}_{2}=\mathrm{R}_{3}=\mathrm{H}, \mathrm{R}_{4}=$ coumaroyl- (92)<smiles>O=C(O)/C=C/c1ccc(O)cc1</smiles>

p-Coumaric acid (93)

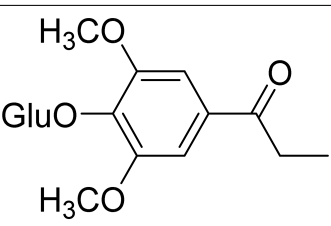

$1-[4-(\beta-\mathrm{D}-$

Glucopyranosyloxy)-3,5dimethoxyphenyl]propanone<smiles>COC(=O)/C=C/c1ccc(O)c(O)c1</smiles>

Caffeic acid $\mathrm{R}=\mathrm{H}$ (94)

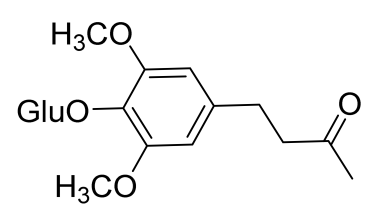

4-(4'-O- $\beta$-D-Glucopyranosyl-3', 5' dimethoxyphenyl)-2butanone

(97)<smiles>COc1cc(/C=C/C(=O)O)ccc1O</smiles>

Ferulic acid (95)<smiles>O=C(O)c1ccccc1O</smiles>

Salicylic acid (97a) 
Another study in the fresh vegetative parts (stems and leaves) of A. cretica, collected in Tunisia, yielded a chlorine-containing sesquiterpene lactone $(2 \beta, 3 \beta$-epoxy- $1 \alpha, 10 \alpha$ dihydroxy-4-chloroguaian-6 $\alpha, 12$-olide) (115) together with two epimeric seco-guaianolides of tanaphillin $(\mathbf{1 2 9}, \mathbf{1 3 0})$ [61]. An additional chlorine-containing guaianolide was obtained from A. ligustica (collected on the Aeolian islands of Italy), namely $3 \beta$-chloro- $4 \alpha, 10 \alpha$ dihydroxy- $1 \alpha, 2 \alpha$-epoxy- $5 \alpha, 7 \alpha$ H-guaia-11(13)-en-12,6 $\alpha$-olide (113), along with iso-secotanapartholide (123) and matricarin (101) [35].

Two uncommon types of Diels-Alder sesquiterpene dimers, achillinin B (118) and achillinin C (119), were isolated from A. millefolium, in which the structures of the monomers of achillinin A and iso-seco-tanapartholide (123) were linked together with $\mathrm{C}-\mathrm{C}$ bonds. It is worth mentioning that achillin $\mathrm{A}$, as a monomer, was also elucidated for the first time in A. millefolium $[64,66]$. Another exploration of the flowers of this plant yielded ten 1,10 -secoguaianolides, including two dimers. Among them, the dimeric compounds were reported as millifolide $A(\mathbf{1 2 0})$ and millifolide $B(\mathbf{1 2 1})$, while millifolide $C(\mathbf{1 1 7})$, a compound with a rare guaianolide rearranged carbon skeleton, was also isolated together with isoseco-tanapartholide (123), arteludovicinolide A (122), 3-acetyl-iso-seco-tanapartholide (125), 3-methoxy-tanapartholide (124), seco-tanapartholide A (126), seco-tanapartholide B (127) and 5-epi-seco-tanapartholide A (131) [64].

Table 7. Sesquitepene lactones from Achillea spp.

\begin{tabular}{|c|c|c|}
\hline Species & Compound & Ref \\
\hline \multirow[t]{2}{*}{ A. alpina $\mathrm{L}$. } & Achillin (103) & {$[18]$} \\
\hline & $4 \beta, 10 \alpha$-Dihydroxy-5 $\beta, 7 \beta, 8 \beta \mathrm{H}$-guaia- & \\
\hline \multirow{3}{*}{ A. biebersteinii Afan. } & 1,11(13)dien-12,8 $\alpha$-olide (116) & \\
\hline & Micranthin(139) & [24] \\
\hline & Sintenin (136) & \\
\hline \multirow{5}{*}{ A. biebersteinii Afan. } & Rupicolin A (98) & \\
\hline & Rupicolin B (99) & [59] \\
\hline & Apressin (106) & \\
\hline & $9 \alpha$-Acetoxycanin $(\mathbf{1 1 1})$ & \\
\hline & 3- $\beta$-Methoxy-iso-seco-tanapartholide (124) & \\
\hline \multirow[t]{6}{*}{ A. clavennae $\mathrm{L}$. } & $\begin{array}{l}3 \beta, 9 \beta \text {-Diacetoxy-1 } \beta \text {-hydroperoxy- } 6 \beta, 7 \alpha \mathrm{H} \text { - } \\
\text { germacra-4,10(14),11(13)-trien-12,6 } \alpha \text {-olide }(137)\end{array}$ & {$[60]$} \\
\hline & 3,9-Diacetoxy-1-hydroxy- $6 \beta, 7 \alpha, 11 \mathrm{H}$-germacra- & \\
\hline & $4,10(14)$-dien-12,6 $\alpha$-olide (138) & \\
\hline & Sintenin (136) & \\
\hline & 3-Acetylridentin (141) & \\
\hline & Artecalin (140) & \\
\hline \multirow{5}{*}{ A. coarctata Poir. } & Arteludovicinolide A (122) & \\
\hline & 11,13- Dihydrodesacetylmatricarin (100) & [7] \\
\hline & Rupicolin A (98) & \\
\hline & Rupicolin B (99) & \\
\hline & $2 \beta, 3 \beta$-Epoxy-1 $\alpha, 10 \alpha$-dihydroxy-4-chloroguaian- & \\
\hline \multirow{3}{*}{ A. cretica L. } & $6 \alpha, 12$-olide $(\mathbf{1 1 5})$ & {$[6]$} \\
\hline & Tanaphillin ( $2 \alpha$ epimer) (129) & {$[6$.} \\
\hline & Tanaphillin (2 $\beta$ epimer) (130) & \\
\hline \multirow[t]{5}{*}{ A. falcata L. } & 3- $\beta$-Methoxy-iso-seco-tanapartholide (124) & [62] \\
\hline & Chrysartemin B (109) & \\
\hline & $1 \beta, 2 \beta$-Epoxy-3 $\beta, 4 \alpha, 10 \alpha$-trihydroxyguaian- & \\
\hline & $6 \alpha, 12$-olide $(\mathbf{1 0 5})$ & \\
\hline & 8-Hydroxy-3-methoxy-iso-seco-tanapartholide & \\
\hline \multirow[t]{5}{*}{ A. falcata L. } & $(132)$ & [63] \\
\hline & 3- $\beta$-Methoxy-iso-seco-tanapartholide (124) & \\
\hline & Iso-seco-tanapartholide (123) & \\
\hline & Rupin A (110) & \\
\hline & Tanaphillin (128) & \\
\hline
\end{tabular}


Table 7. Cont.

\begin{tabular}{|c|c|c|}
\hline Species & Compound & $\operatorname{Ref}$ \\
\hline A. ligustica All. & $\begin{array}{l}3 \beta \text {-Chloro- } 4 \alpha, 10 \alpha \text {-dihydroxy- } 1 \alpha, 2 \alpha \text {-epoxy- } \\
5 \alpha, 7 \alpha \text { H-guaia- } 11(13) \text {-en- } 12,6 \alpha \text {-olide }(\mathbf{1 1 3}) \\
\text { Iso-seco-tanapartholide }(\mathbf{1 2 3}) \\
\text { Matricarin (101) }\end{array}$ & [35] \\
\hline A. millefolium $\mathrm{L}$. & $\begin{array}{l}\text { 3-Acetyl-iso-seco-tanapartholide (125) } \\
\text { Arteludovicinolide A (122) } \\
\text { 5-Epi-seco-tanapartholide A (131) } \\
\text { Iso-seco-tanapartholide (123) } \\
\text { 3-Methoxy-tanapartholide (124) } \\
\text { Millifolide A (120) } \\
\text { Millifolide B (121) } \\
\text { Millifolide C (117) } \\
\text { Seco-tanapartholide A (126) } \\
\text { Seco-tanapartholide B (127) }\end{array}$ & [64] \\
\hline A. millefolium L. & Achillinin A (104) & [65] \\
\hline A. millefolium $\mathrm{L}$. & $\begin{array}{l}\text { Achillinin B (118) } \\
\text { Achillinin C (119) }\end{array}$ & {$[66]$} \\
\hline A. millefolium $\mathrm{L}$. & $\begin{array}{l}\text { Achillin (103) } \\
\text { Leucodin (102) }\end{array}$ & [67] \\
\hline A. wilhelmsii K. Koch. & $\begin{array}{l}\text { 1 } \beta, 10 \beta \text {-Epoxydesacetoxymatricarin }(\mathbf{1 0 7}) \\
\text { Leucodin }(\mathbf{1 0 2})\end{array}$ & {$[50]$} \\
\hline A. wilhelmsii K. Koch. & $\begin{array}{l}\text { Artecanin (109) } \\
\text { Artecaninhydrate (108) } \\
\text { Artemargyinolide B (112) } \\
\text { Chloroklotzchin (114) } \\
\text { Deacetylmatricarin-8-O-b-glucopyranoside (104) } \\
\text { Hanphyllin (134) } \\
\text { Leucodin (102) } \\
\text { Wilhelmsin (133) } \\
\text { Wilhelmsolide (135) }\end{array}$ & {$[51]$} \\
\hline
\end{tabular}

Table 8. Chemical structures of Guaianolides from Achillea spp. Part I.

\begin{tabular}{|c|c|c|c|}
\hline$=$ & $=\mathrm{R}_{2}$ & $\mathbf{R}_{\mathbf{1}}$ & $\mathbf{R}_{2}$ \\
\hline Rupicolin A $\Delta^{9(10)}(\mathbf{9 8})$ & $\begin{array}{c}\text { 11,13-Dihydrodesacetylmatricarin } \\
(\mathbf{1 0 0})\end{array}$ & $\begin{array}{c}\alpha \mathrm{OH} \\
\beta \mathrm{H}\end{array}$ & $\mathrm{CH}_{2}$ \\
\hline \multirow[t]{4}{*}{ Rupicolin B $\Delta^{10(14)}(99)$} & Matricarin (101) & $\begin{array}{c}\alpha \mathrm{OAc} \\
\beta \mathrm{H}\end{array}$ & $\begin{array}{c}\alpha \mathrm{CH}_{3} \\
\beta \mathrm{H}\end{array}$ \\
\hline & Leucodin (102) & $\mathrm{H}, \mathrm{H}$ & $\begin{array}{c}\alpha \mathrm{CH}_{3} \\
\beta \mathrm{H}\end{array}$ \\
\hline & Achillin (103) & $\mathrm{H}, \mathrm{H}$ & $\begin{array}{c}\alpha \mathrm{H} \\
\beta \mathrm{CH}_{3}\end{array}$ \\
\hline & $\begin{array}{l}\text { Deacetylmatricarin-8-O- } b \text { - } \\
\text { glucopyranoside } \\
(\mathbf{1 0 4})\end{array}$ & O-Glc & $\begin{array}{c}\alpha \mathrm{CH}_{3} \\
\beta \mathrm{H}\end{array}$ \\
\hline
\end{tabular}


Table 9. Chemical structures of Guaianolides from Achillea spp. Part II.

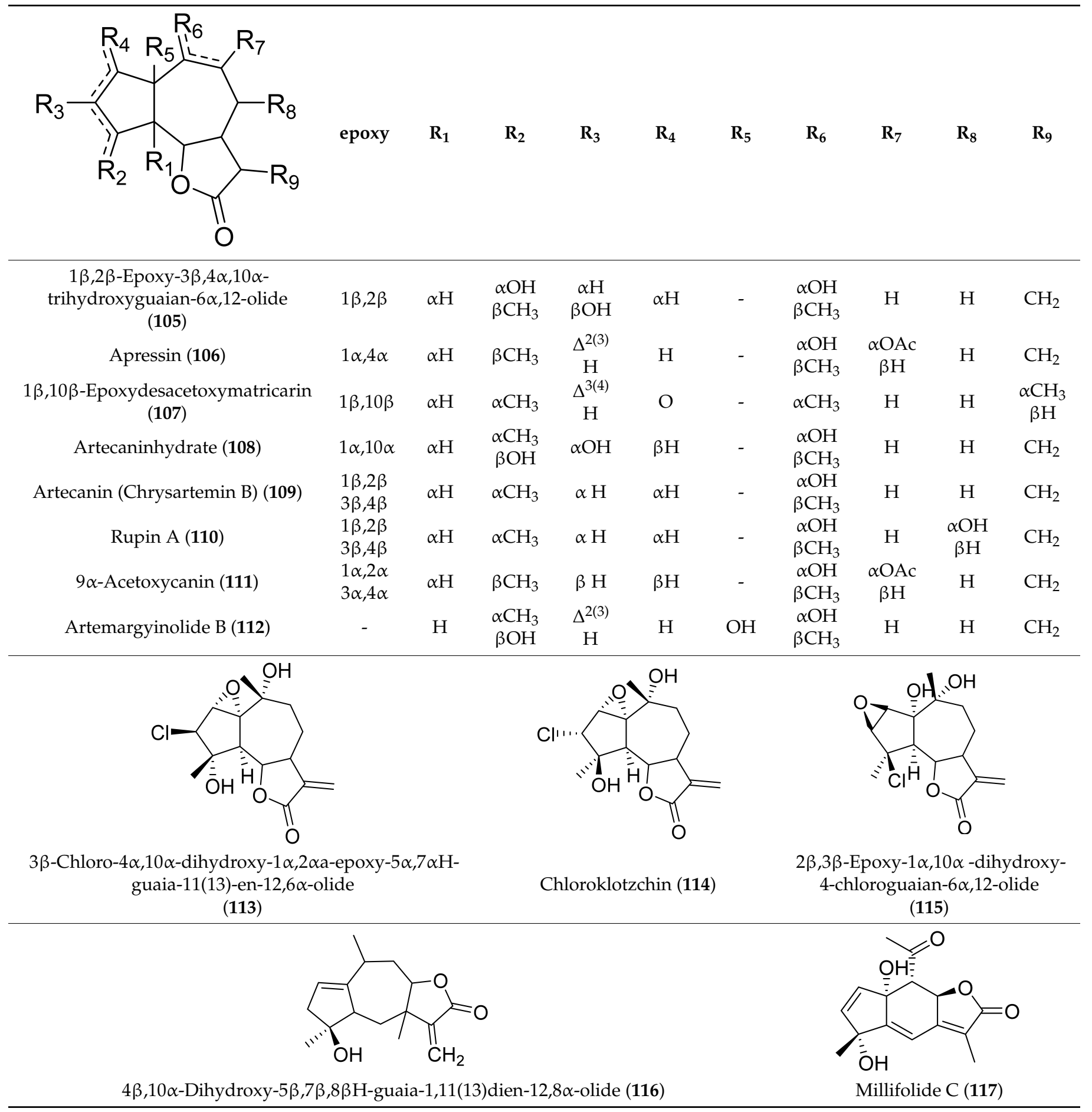


Table 10. Chemical structures of sesquiterpene lactone dimers from Achillea spp.

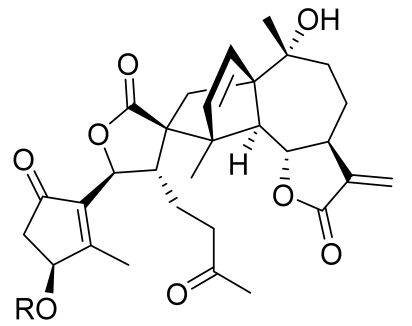

Achillinin B (118) $\mathrm{R}=\mathrm{CH}_{3}$ Achillinin C (119) R = H

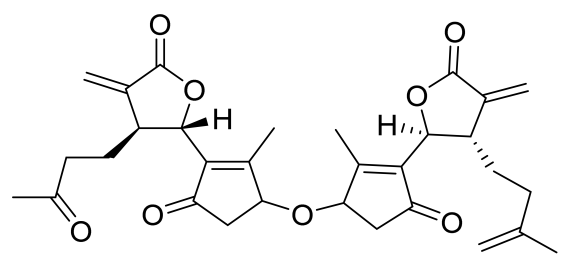

Millifolide A (120)

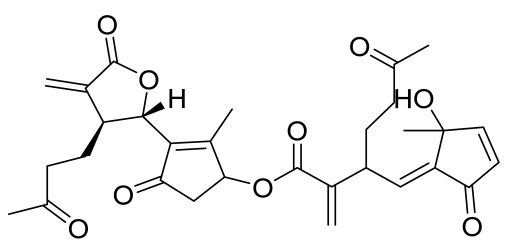

Table 11. Chemical structures of seco-guaianolides from Achillea spp.

(12)


Table 11. Cont.

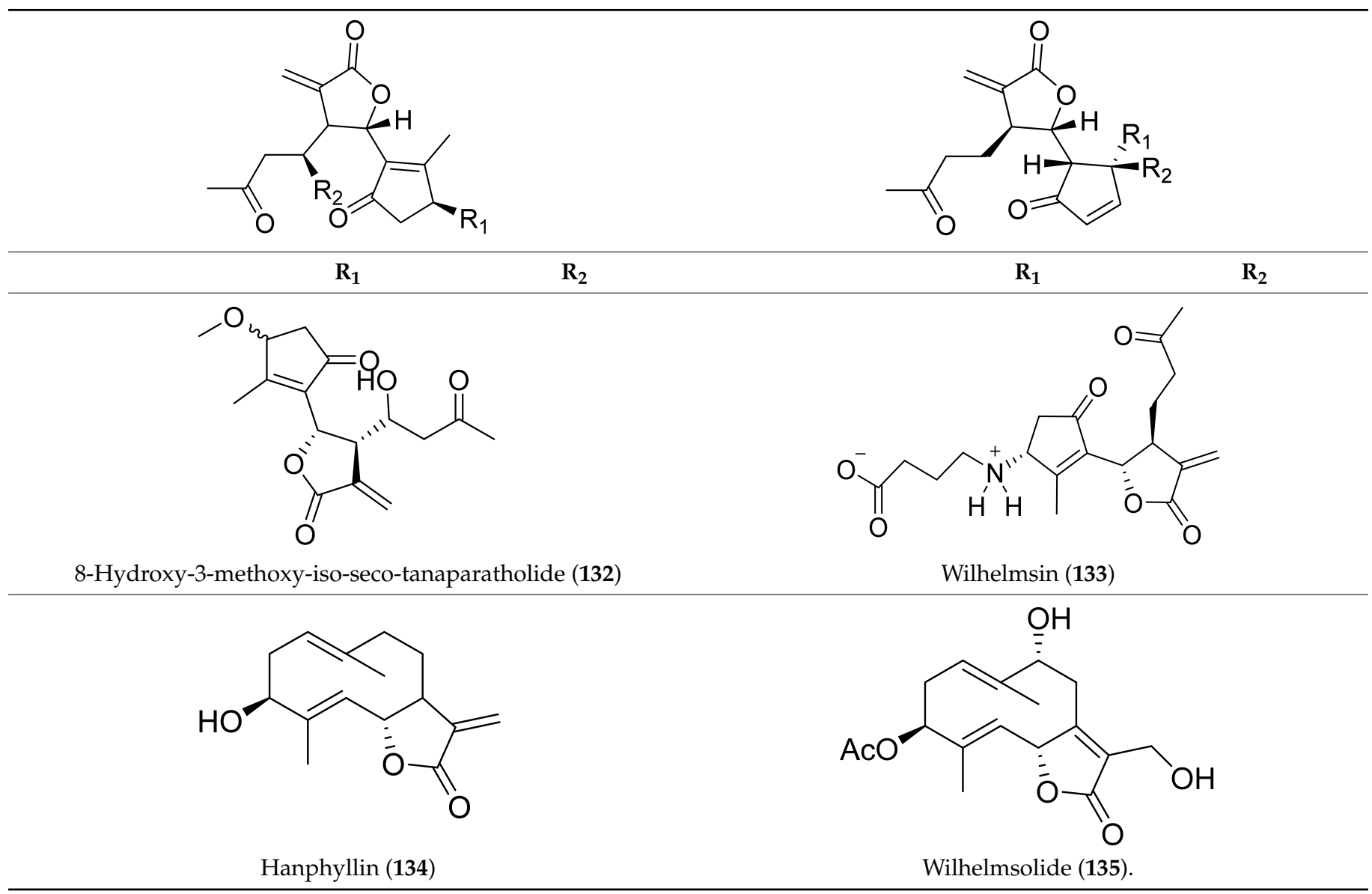

Table 12. Chemical structures of germacranolides from Achillea spp.

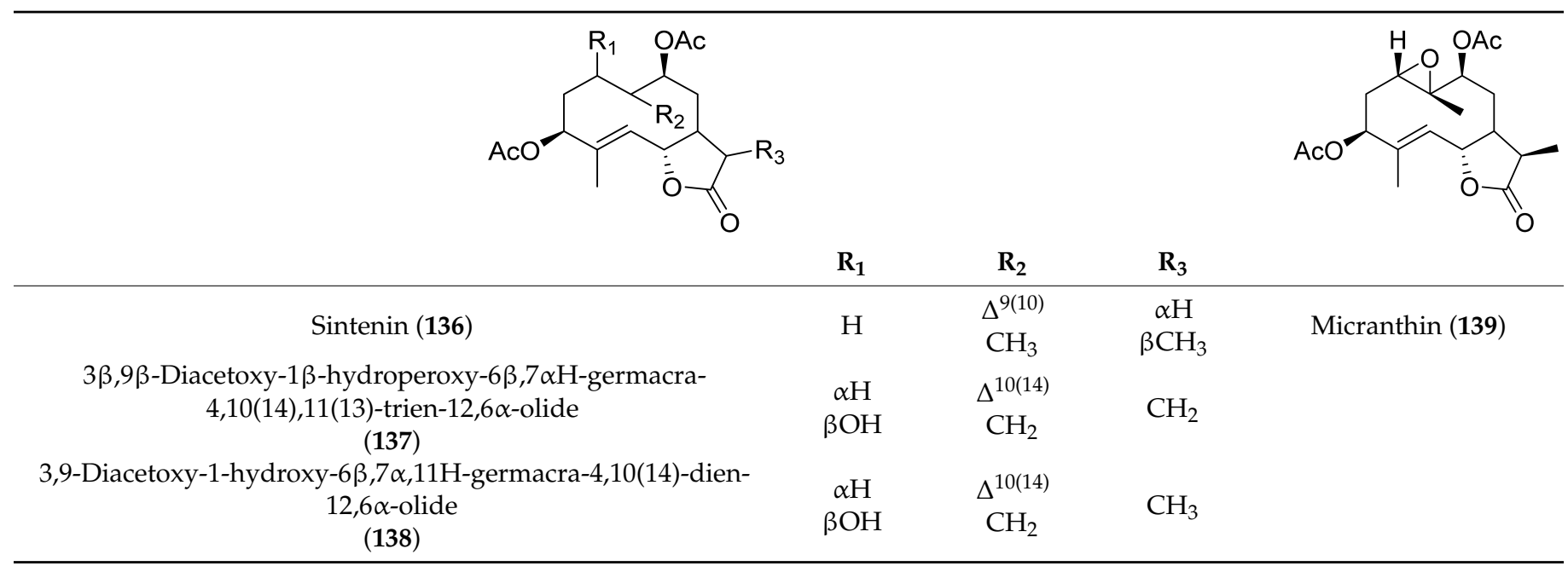


Table 13. Chemical structures of eudesmanolides from Achillea spp.

Artecalin (140)

\subsubsection{Other Compounds}

In addition to the principal components of the genus, such as the sesquiterpene lactones and the flavonoids, several phytochemical investigations have revealed the presence of other compounds. More specifically, some studies report the isolation of alkamides [68], lipid acids [27,40] and sterols [31]. Saponins have been also isolated, for example from A. wilhelmsii [65]. Interestingly, even from the well-investigated A. millefolium, two new compounds were recently isolated, one terpenoid and one lignan [69]. These indicate that the phytochemical profile of the taxa could be subjected to variations; however, this biodiversity may explain the bioactivity of Achillea species.

\section{Ethnopharmacological Relevance: Traditional Uses of the Genus Achillea and Established Biological Effects}

The genus Achillea encompasses a significant number of medicinal plants globally. It is nevertheless noteworthy that traditional practices are diverse with regional and cultural differences and similarities. In a broad context, the various Achillea species are used for the prevention and treatment of different diseases, mainly in the form of infusions and decoctions (herb, flowers and leaves) for spasmodic gastrointestinal and hepato-biliary disorders, haemorrhages, pneumonia, rheumatic pain, wound healing, as diuretic, antiinflammatory and antipyretic agents, and to treat menstrual and gynaecologic abnormalities. Due to these activities, various thematic names have been given to the genus such as: bloodwood, staunchweed, militaryherb, etc. [15].

The following section summarizes the reported ethnopharmacological uses of 24 Achillea species, with the aim to bridge these features with current studies and in fact to provide some form of conclusive evidence and discuss the associated biological and pharmaceutical properties. Moreover, details on the uses of these species and the associated references are indicated in Table 14 [7,8,18-20,28,30,31,35,36,44,49,50,56,57,60,67,70-91].

The aerial parts of A. aleppica are used in Turkey under the name "Civanperçemi" in decoctions due to their diuretic, carminative, emmenagogue, antiasthmatic, cardiotonic, stomachic, and tonic effects, as well as for colds, nephralgia, gynaecologic ailments and wound healing $[70,71]$. Concerning its biological activity, the methanol extract of $A$. aleppica subsp. zederbaueri was tested on cell viability and proliferation of the MCF7 (breast cancer cell line) by the 3-(4,5-dimethylthiazol-2-yl)-2,5-diphenyltetrazolium bromide (MTT) assay. Since at concentrations of 100 and $250 \mu \mathrm{g} / \mathrm{mL}$ it effectively inhibited the cell proliferation, it could be considered as a herbal-based anti-cancerous agent. Another study investigated the protective ability of the ethanol extracts of A. aleppica subsp. aleppica and A. aleppica subsp. zederbaueri against the lipid peroxidation, protein oxidation and DNA damage induced by the Fenton system. This study revealed the antioxidant properties of the extract, which could contribute to the prevention of ROS to related chronic inflammatory diseases, such as cardiovascular diseases and cancer [92]. Moreover, Bariş et al. [93] determined noticeable antimicrobial activity and confirmed the strong antioxidant activities of the above-mentioned extracts.

A. alpina is widely distributed in Asia and North America. It was recorded in Korea as a folk medicine for stomach disorders [18], while it was reported in the Pharmacopoeia of the People's Republic of China as having many properties, such as detox, dampness- 
clearing, bloodstream promotion, and analgesic activities [57]. Another report suggests its use as a traditional herbal medicine for the treatment of acute inflammation and as healthpromoting agent (teas and spice) [15]. Pharmacological studies showed that it possesses anti-inflammatory, antipyretic, and sedative properties [15]. According to Lee et al. [18], two compounds isolated from this plant were highly active, and methyl 3,5-dicaffeoylquinic acid (78) in particular showed strong antioxidant activities, while isovitexin (11) showed antimelanogenic activity that was associated with the downregulation of intracellular tyrosinase activity, indicating that such compounds could act as antihyperpigmentation agents for skincare products. Moreover, a pair of natural caffeoylquinic acid derivatives (isochlorogenic acids A (86) and B (87) isolated from the plant showed an inhibitory activity (with antagonistic effects) on the transient receptor potential vanilloid 3 (TRPV3) channel by using a calcium fluorescent assay. This channel is associated with numerous human skin diseases including skin inflammation, cutaneous pain, chronic itch, atopic dermatitis, and Olmsted syndrome [57,94].

In Mongolia, A. asiatica has been traditionally used for its intestinal and stomach antiinflammatory effects, for persistent fever, ulcers, wounds, and rheumatism [20]. Recently, the ethanol extract of this plant was tested on cutaneous wound healing in vivo (rats), and the study showed the beneficial effect of the extract in wounded skin, by supporting the keratinocyte development [20].

A. atrata has been claimed to be effective in respiratory tract disorders [72]. In recent years, the non-polar extract, as well as the isolated compounds of A. atrata subsp. multifida, were examined for their antimicrobial effects in vitro. The extract was active against Candida albicans and Bacillus subtilis, while santin (39), centaureidin (49), apigenin (2), and its 7-O- $\alpha$-glucoside (8) were active against B. subtilis and C. albicans, while santin (39) and apigenin (2) exhibited activity against Escherichia coli [95].

Another study reported the uses of A. bibersteinii, found in Turkey, as a diuretic for wound healing and for the treatment of gastrointestinal disorders, including abdominal pains and haemorrhoids [70]. Moreover, in Iran, A. biebersteinii (local name: ormaderen) has been used for colds, nephralgia, gynaecologic conditions, such as women' sterility and as emmenagogue or for jaundice, and as astringent for skin conditions, such as oedema and erythema $[76,77]$. The antioxidant, antimicrobial and enzyme inhibitory abilities of this plant along with two other Achillea spp. (A. millefolium, A. teretifolia) were assessed for different extracts. In the Ferric Reducing Antioxidant Power (FRAP) assay, the best result was obtained from the A. biebersteinii MeOH extract (196.12 mgTE/g extract), whilst in the CupricReducing Antioxidant Capacity (CUPRAC) assay, the most potent reducing capacity was obtained for the A. millefolium $\mathrm{MeOH}$ extract (255.66 mgTE/g extract). The $\mathrm{MeOH}$ and $\mathrm{H}_{2} \mathrm{O}$ extracts had stronger antioxidant effects. Apparently, the EtOAc extract of A. teretifolia was more effective, followed by the EtOAc extract of A. millefolium and A. biebersteinii, on the assessed cholinesterases, while the highest tyrosinase inhibitory effect was detected for $\mathrm{MeOH}$ extract (34.24 mg KAE/g). Moreover, the EtOAc and $\mathrm{MeOH}$ extracts exhibited remarkable antibacterial (MICs $0.015-0.60 \mathrm{mg} / \mathrm{mL}$, MBCs $0.025-0.8 \mathrm{mg} / \mathrm{mL}$ ) and antifungal $(0.015-0.4 \mathrm{mg} / \mathrm{mL}$, MBCs $0.025-0.8 \mathrm{mg} / \mathrm{mL}$ ) potential [96]. In accordance with the previous results, another study revealed the antioxidant and antimicrobial properties of $A$. biebersteinii extracts. Concerning the wound-healing property of this plant, in vivo studies gave an account by testing different extracts on mice and rats, suggesting that the n-hexane extract possesses higher activity [97]. Of note, rupicolin A (98) and rupicolin B (99) isolated from this plant, showed to have promising anti-inflammatory effects ( $76 \%$ and $80 \%$ inhibitions, respectively) by inhibiting the lipopolysaccharide-induced NO production in RAW264.7 macrophage cells [59]. To provide more evidence in accordance with the traditional application of $A$. biebersteinii, the analgesic, antidepressant and anxiolytic activities of this plant were studied in vivo, showing that the methanol extract exerted analgesic and anxiolytic, but not antidepressant activities [98].

In Turkey, the leaves of A. cappadocica under the local name of "Buyucan" were used externally as a powder, or internally as a decoction/infusion for its astringent, emmena- 
gogue, stomachic effects and to treat oedema [71]. The antioxidant, anticholinesterase, and antimicrobial activities of different extracts were tested, indicating that the methanol and water extracts had higher cation radical scavenging activity than that of $\alpha$-tocopherol and BHT at $100 \mu \mathrm{g} / \mathrm{mL}$ in (2,2' -azino-bis(3-ethylbenzothiazoline-6-sulfonic acid (ABTS) method. The acetone extract showed $70.62 \%$ inhibitory activity against butyrylcholinesterase at $200 \mu \mathrm{g} / \mathrm{mL}$, while the acetone and methanol extracts exhibited moderate antimicrobial activity [99].

A. coarctata is locally used in the area of Kozani, central Greece, as a traditional remedy for gastrointestinal ailments and hypertension, due to its diuretic effects [7]. Moreover, this plant was also reported from the neighbouring country, Turkey, where it has been used under the common name "Civanperçemi" for its diuretic, carminative, and menstrual effects in the form of decoction (aerial parts) [71]. Recently, extracts and isolated compounds from this plant showed some significant cytotoxic effects in vitro against the human cancer cell lines MCF-7 and HeLa [7].

For A. collina, found in Bosnia and Herzegovina, contradictory uses have been reported, i.e., for bedwetting by children and to increased diuresis and excretion of urinary stones. Moreover, it has been used for blood purification, skin conditions such as injuries, rashes and psoriasis, purulent ulcers, liver ailments, regulation of menstruation, bronchitis, asthma and throat ache [72,73]. Infusions from this plant showed good antioxidant and cytoprotective properties in vitro [100], as well as potent analgesic activity in vivo [101].

A. cretica is among the several Achillea species that are used traditionally for the treatment of gynaecological disorders and pathological symptoms analogous to endometriosis according to Persian (Iranian) traditional medicine, as well as for its wound-healing properties and to treat urogenital and respiratory disorders [28]. With the aim to investigate the traditional use of this plant, Bina et al. [28] reported that the plant's extract could be used for the management of endometriosis in vivo by modulating inflammatory cytokines.

Nowadays, A. clavennae is used in Croatian folk medicine to relieve ailments such as abdominal pain, common cold, influenza, and respiratory disorders. However, it was described earlier at the beginning of the 17th century as a healing plant with cholagogue, stomachic, and anthelmintic properties [77]. Isolated compounds from the plant were tested for their antiproliferative action with regard to HeLa, K562 and Fem-X human cancer cell lines. The most active compound was centaureidin (49) followed by $9 \alpha$-acetoxyartecanin and apressin (106), with significant cytotoxic effects exerted on all tested lines, while inducumenone exhibited moderate activity [77]. In another in vitro study, four extracts of Achillea species were tested, and A. clavennae in particular showed sufficient evidence for its ethnopharmacological use, possessing a broad spectrum of antimicrobial activities against many tested strains [102].

A. falcata is a well-known medicinal plant for local people in Jordan, with beneficial effects on internal haemorrhages, uterine haemorrhoids, stomach ailments, gastritis, and bladder stones [56,78]. An antimicrobial investigation of A. falcata indicated that extracts possess low antibacterial activity in comparison with other Achillea species [103]. Though, another study reported that the hydro-alcoholic extract had bactericidal activity against Streptococcus pneumoniae, Bacillus cereus and Klebsiella pneumoniae rather than bacteriostatic effect. Another study showed no significant activity against Gram-negative bacteria and Candida albicans [104].

A. filipendulina, found in Israel, is one of the 15 plant species that are used to treat hypoglycaemia [56]. It is native to central and southwestern Asia, commonly called "Fernleaf Yarrow" or "Bumadaran Zard" or "Sari Chichak" by locals and is used to treat various conditions. More precisely, decoctions of this plant were used to treat arthritis, cardiovascular diseases, congestions, gastrointestinal disorders, gout, and malaria, and as a diuretic, anthelmintic, and purgative agent [79]. Antioxidant and antimicrobial effects have been reported for this plant $[22,105]$.

A. fragrantissima, found in Israel, is another species used to treat hypoglycaemia [56]. In addition, the plant was used by Bedouin communities in Egypt in various conditions, 
including gastrointestinal disturbances, eye infections, and smallpox [30]. In Egypt, there are several reports on the declared folk and traditional uses of this species to treat fever caused by viral infection, as well as in chronic diseases, such as arthritis and diabetes [80]. Moreover, in the folk medicine of the Middle East, this plant is reputed for its antidiabetic properties [31], and more specifically, in the Arabia region, A. fragrantissima is used for the treatment of respiratory diseases and gastrointestinal disturbances [81]. Additionally, cirsiliol (22) isolated from A. fragrantissima caused the relaxation of contracted rats' proximal aortas, tracheas, urinary bladders, and uteruses [106]. Furthermore, the ethanolic extract of the plant was tested for its anti-inflammatory effects on lipopolysaccharide-activated primary cultures of brain microglial cells, suggesting that phytochemicals from this extract could be beneficial in preventing/treating neurodegenerative diseases [81]. Moreover, the non-polar and polar extracts possessed anti-inflammatory and analgesic properties comparable to indomethacin and acetylsalicylic acid, respectively, while the extracts attenuated the macroscopic colonic damage induced by acetic acid in rats. Notably, no signs of toxicity were reported in this study [106]. Furthermore, the antioxidant and antimicrobial effects of this plant were confirmed by different pharmacological studies [104].

A. lanulosa has been used in preparations of folk medicines, either internally as a decoction or infusion, or externally as a lotion or ointment, to treat many ailments, such as for wound healing and as a dermatological aid for sprains and swollen tissues, common colds, head- and earache, indigestion problems, and haemorrhoids, as well as a laxative agent, etc. [56]. No recent in vitro or in vivo studies could be obtained regarding $A$. lanulosa.

A. ligustica, found in the Mediterranean region has been used as folk remedy for the treatment of gastrointestinal disorders [82]. In Italy, the species A. ligustica was used as an anthelmintic agent and for the treatment of chronic diseases such as rheumatism and skin disorders or inflammation. Focusing on Sicilia and Sardinia, the fresh leaves of this plant were used as an antimicrobial and haemostatic agent topically applied or per os (swallowed as pellets) against stomach-ache $[35,83,89]$. Strong antioxidant and anti-inflammatory activities $[107,108]$, as well as weak antidiabetic effects [109], have been reported for this plant.

Regarding A. magnifica, an endemic plant of Turkey known as the "karcivanı", its dried flowering parts are mixed with honey and used to treat stomach ailments [35,36,89]. Taskin et al. [37] investigated the antioxidant, antiurease, anticholinesterase, and antiproliferative activities of different $A$. magnifica extracts. This study revealed that the chloroform crude extract or prepared with chitosan nanoparticles, as well as the isolated compounds (elenolic acid, luteolin (3), and eupatilin (23a)), showed strong antioxidant and cytotoxic activities. In fact, chitosan nanoparticles not only showed a strong and selective cytotoxic activity in breast cancer cells, but also did not have toxic effects in normal cells, suggesting that it might be suitable for preclinical and clinical studies in cancer treatment. Moreover, the ethanol extract has strong antiurease and anticholinesterase potential [37].

Another species from Italy, $A$. moschata, is also used in traditional medicine both in human and veterinary health-related problems. It is administered as an infusion against dyspepsia, abdominal bloating, flatulence, and gastric pains and, in general, to benefit the digestive system, as well as for colds, coughs, dysmenorrhea, earache, fever, gout, headache, hypertension, insomnia, menopausal disorders, neuralgia, oliguria, skin inflammations, urinary tract inflammations and vaginitis [43]. Moreover, it is traditionally used against bacterial infections, and some in vitro studies demonstrated sufficient evidence for this ethnopharmacological indication. In fact, extracts from the aerial parts were found to possess a broad spectrum of antimicrobial activity against both Gram-positive and Gram-negative bacteria, showing that the less polar extracts possess greater efficacy than the methanolic extracts. Apparently, the methanolic extract was significantly effective against synthetic radicals (ABTS ${ }^{+}$and $\left.\mathrm{DPPH}\right)[43,44]$.

Among the various species of the genus Achillea, A. millefolium stands out and is extensively used throughout the world. It is worth mentioning that the name millefolium reflects to 1000 leaves showing its folios branches. Documented by many sur- 
veys in the literature, yarrow has been extensively used against inflammatory disorders and wound healing [20,110], or for spasmodic gastrointestinal disorders and appetiteenhancing drugs due to its bitter taste, or for hepato-biliary complaints, as well as for many gynaecological abnormalities, including the regulation of the menstrual cycle, its emmenagogue properties or pathological symptoms similar to endometriosis in traditional medicine. The aerial parts of the plant are generally applied as aqueous or alcoholic extracts [111,112]. Herba millefolii is an official traditional herbal medicinal product used for the temporary loss of appetite, the symptomatic treatment of mild, spasmodic gastrointestinal complaints, including bloating and flatulence, the symptomatic treatment of minor spasms associated with menstrual periods and for the treatment of small superficial wounds [10]. Around the world, such as in Middle Europe (e.g., Germany, Italy, Hungary), A. millefolium is listed among the most commonly used medicinal plants. Its use against bleeding, stomach complaints, and menstrual spasm, both as an infusion or externally as a poultice, suggests its medicinal importance. Moreover, in Serbia, it is most frequently used for gastrointestinal ailments such as infusion. Another study reported its use to treat various complaints including gastrointestinal, urinary, respiratory, and dermatological disorders [84]. Moreover, in Russian Pharmacopeia, A. millefolium has been recorded as a haemostatic and anti-inflammatory agent [85]. In traditional Persian medicine, it has been claimed to have anti-inflammatory and emmenagogue properties and is used for the regulation of the menstrual cycle and treatment of gynaecological disorders, including endometriosis, as well as for the treatment of urogenital and respiratory disorders and as a wound-healing agent [28]. Moreover, in West Azerbaijan, the infusion of dried flowers is considered suitable for the treatment of haemorrhoids, dyspepsia, dysmenorrhoea and gastritis [86]. Similarly, in West Himalaya, India, aerial parts are used for gastric problems and fever [86]. Once more, in Unani medicine of India, A. millefolium is used due to its anti-inflammatory, emmenagogue, antipyretic, diuretic, and analgesic properties [88]. Its medical activities are also involved in many traditional practices in Turkey, mainly applied for the treatment of wounds, cold, influenza and for stomach or urinary problems, as antiseptic or antitussive agents, for abdominal pain, etc. [56,71].

It is worth mentioning that veterinary use of the Achillea spp. is rarely recorded. However, in Iran, A. nobilis was used against animal parasites, skin wounds and infections [90]. Taskin et al. [45] investigated the antioxidant, antiurease and antimicrobial activities of different extracts from $A$. nobilis subsp. neilreichii. This study revealed that some of the extracts possess significant antimicrobial and antioxidant activities.

A. santolina, in Iraq, is used to treat hypoglycaemia [56], while in Jordan, the traditional medical usage of this plant is based on its carminative, antispasmodic, depurative properties, as well as for its effects on stomach-aches and diabetes. The hypoglycaemic effects of the plant have been examined both in vitro and in vivo. The study of Kasabri et al. [113] showed that $A$. santolina lacked any favourable in vitro anti- $\alpha$-amylase and anti- $\alpha$-glucosidase effects, while Yazdanparast et al. [114] showed that it has a high hypoglycaemic activity against pancreatic damage in streptozotocin (STZ)-treated diabetic rats, which could be attributed to its antioxidative potential.

Once again in Turkey, a member of the Achillea genus, namely A. setacea (local name: Ortkesan), was used traditionally as an emmenagogue and stomachic [71]. Rupicolin B (99) and 11,13-dehydrodeacetylmatricarin isolated from this plant showed antiphlogistic/antiinflammatory effects in the croton oil ear test [115].

A. tenuifolia (local name: Çoban kirpiği) has been used internally as a decoction for the treatment of hypercholesterolemia, diabetes, asthma, bronchitis and cough [71]. A recent study by Bagheri et al. investigated the hydro-alcoholic extract of this plant on anxiety-like behaviour and reproductive parameters in a rat model of chronic restraint stress [116].

Furthermore, A. vermicularis, known by the local name of "Civanperçemi", is used in a form of decoction for its stomachic problems [71]. Other reports, especially in Iranian traditional medicine, suggest its antitumour effect. Based on this, the apoptosis induction 
ability was evaluated through the activation of caspase 3 in intact MCF-7 cells, suggesting that the extract demonstrated signs of caspase 3 activations [117]. More recently, studies reported its antioxidant, anti-inflammatory and antiurease potential [49].

A. wilhelmsii has been used traditionally used for the treatment of gastrointestinal and pulmonary complaints in Italy and Turkey [91]. The capitula of this plant were traditionally used as a diuretic, for abdominal pain, as stomachic, emmenagogues and to treat women's sterility, while the aerial parts were used as antihaemorrhoidal agents [71]. Lastly, in Iran (local name "boomaderun"), it was used for body- and stomach- ache, blood coagulation, diabetes, hypertension, kidney stones and constipation [50,71]. Several studies report the antioxidant [118], as well as the antimicrobial effects of this plant $[118,119]$. Niazmand et al. [120] investigated the traditionally reported gastrointestinal effects on rats gastric acid output in basal, vagotomized, and vagal-stimulated conditions, suggesting that the $A$. wilhelmsii extract exhibits an inhibitory effect on the acid output in basal conditions. Moreover, another work reports that the extracts of $A$. wilhelmsii act as calcium antagonists and the intestinal relaxation effect of this plant supports its traditional use as an antispasmodic [121]. Regarding the traditional uses for pain relief, a recent study reports the antinociceptive activity of the plant by using the hot-plate test in BALB/c mice [122].

Natural products and traditional medicines are of great importance, attracting the attention of the scientific community, and affording a plethora of bibliographic data. Though, valuable information is missing not only for the reliability of these ethnopharmacological reports but also in many cases where the mechanisms of action from validated traditional uses are not currently understood. Taking into consideration the above section on the ethnopharmacological relevance of the traditional uses of 24 Achillea spp. in combination with modern results for their biological activities, we can conclude that up to now mainly in vitro and in vivo experiments have been conducted, but clinical trials are limited.

The utilization of extracts or/and isolates from Achillea plants seems to have potential as antioxidant, antimicrobial, anti-inflammatory, cytotoxic agents, as well as for wound healing and the treatment of other skin-related ailments, endometriosis, neurodegenerative diseases, diabetes, and pain, while no significant toxicity was observed.

Table 14. Traditional medicinal uses of Achillea spp.

\begin{tabular}{|c|c|c|}
\hline Species & Traditional Uses & Ref. \\
\hline A. aleppica DC. & $\begin{array}{l}\text { Diuretic, carminative, emmenagogue, antiasthmatic, cardiotonic, stomachic, } \\
\text { tonic effects, for colds, nephralgia, gynaecologic ailments and wound healing }\end{array}$ & {$[70,71]$} \\
\hline \multirow{2}{*}{ A. alpina $\mathrm{L}$. } & Stomach disorders & [18] \\
\hline & Detox, clearing dampness, bloodstream promotion, and analgesic & [57] \\
\hline A. asiatica Serg. & $\begin{array}{l}\text { Intestinal and stomach disorders, persistent fever, ulcers, wounds, } \\
\text { inflammations and rheumatism }\end{array}$ & {$[20]$} \\
\hline A. atrata L. & Respiratory disorders & [72] \\
\hline \multirow[t]{2}{*}{ A. bibersteinii Afan. } & $\begin{array}{l}\text { Diuretic, wound healing, gastrointestinal disorders, including abdominal } \\
\text { pains and haemorrhoids }\end{array}$ & {$[67]$} \\
\hline & $\begin{array}{l}\text { Colds, nephralgia, gynaecologic conditions such as women' sterility, } \\
\text { emmenagogue or jaundice, and astringent for skin conditions such as } \\
\text { oedema and erythema }\end{array}$ & $\begin{array}{l}{[73]} \\
{[74]}\end{array}$ \\
\hline A. cappadocica Hausskn. \& Bornm. & Astringent, emmenagogue, stomachic effects and to treat oedema & [71] \\
\hline A. coarctata Poir. & Gastrointestinal ailments and hypertension, due to its diuretic effects & [7] \\
\hline & Menstrual effects & [74] \\
\hline A. collina Becker ex. Rchb. & $\begin{array}{l}\text { Bedwetting by children and contradictory to increased diuresis and excretion } \\
\text { of urinary stone blood purification, skin condition such as injuries, rashes } \\
\text { and psoriasis, purulent ulcers, liver ailments, regulation of menstruation, } \\
\text { bronchitis, asthma and throat ache }\end{array}$ & {$[75,76]$} \\
\hline A. cretica L. & $\begin{array}{l}\text { Gynaecological disorders and pathological symptoms analogous to } \\
\text { endometriosis, wound healing, urogenital and respiratory disorders }\end{array}$ & [28] \\
\hline A. clavennae $\mathrm{L}$. & Abdominal pain, common cold, influenza, and respiratory disorders & {$[60,77]$} \\
\hline A. falcata L. & $\begin{array}{l}\text { Internal haemorrhage, uterine haemorrhoid, stomach ailment, and bladder } \\
\text { stones }\end{array}$ & {$[56,78]$} \\
\hline
\end{tabular}


Table 14. Cont.

\begin{tabular}{|c|c|c|}
\hline Species & Traditional Uses & Ref. \\
\hline \multirow[t]{3}{*}{ A. filipendulina Lam. } & Hypoglycaemia & [56] \\
\hline & $\begin{array}{l}\text { Arthritis, cardiovascular diseases, congestions, gastrointestinal disorders, } \\
\text { gout, malaria, and as a diuretic, anthelmintic, and purgative agent }\end{array}$ & {$[79]$} \\
\hline & Hypoglycaemia & [56] \\
\hline \multirow[t]{3}{*}{ A. fragrantissima (Forssk.) Sch. Bip. } & $\begin{array}{l}\text { Gastrointestinal disturbances, eye infections, and smallpox, fever caused by } \\
\text { viral infection, chronic diseases, such as arthritis and diabetes }\end{array}$ & {$[30,80]$} \\
\hline & Diabetes & [31] \\
\hline & Respiratory diseases and gastrointestinal disturbances & {$[81]$} \\
\hline \multirow[t]{2}{*}{ A. ligustica All. } & Gastrointestinal disorders & {$[82]$} \\
\hline & $\begin{array}{l}\text { Anthelmintic, against stomach-ache, chronic diseases such as rheumatism } \\
\text { and skin disorders or inflammation, antimicrobial and haemostatic agent }\end{array}$ & {$[35,83]$} \\
\hline A. lanulosa Nutt. & $\begin{array}{l}\text { Wound healing, as dermatological aid for sprains and swollen tissues, } \\
\text { common cold, head and earache, indigestion problems, haemorrhoids and } \\
\text { laxative agent }\end{array}$ & [56] \\
\hline \multirow[t]{7}{*}{ A. millefolium L. } & Bleeding, stomach complaints, menstrual spasm & [8] \\
\hline & Gastrointestinal ailments, urinary, respiratory, and dermatological disorders & [84] \\
\hline & Haemostatic and anti-inflammatory agent & {$[85]$} \\
\hline & $\begin{array}{l}\text { Anti-inflammatory and emmenagogue properties, for regulation of } \\
\text { menstrual cycle and gynaecological disorders including endometriosis, } \\
\text { urogenital and respiratory disorders, and as wound-healing agent }\end{array}$ & [28] \\
\hline & Haemorrhoids, dyspepsia, dysmenorrhoea and gastric problems and fever & {$[86,87]$} \\
\hline & $\begin{array}{l}\text { Anti-inflammatory, emmenagogue, antipyretic, diuretic, and analgesic } \\
\text { properties }\end{array}$ & [88] \\
\hline & $\begin{array}{l}\text { Wounds, cold, influenza and for stomach or urinary problems, as antiseptic, } \\
\text { antitussive, for abdominal pain }\end{array}$ & {$[56,71]$} \\
\hline \multirow[t]{2}{*}{ A. magnifica Hub.-Mor. } & Stomach ailments & {$[36,89]$} \\
\hline & $\begin{array}{l}\text { Dyspepsia, abdominal bloating, flatulence, gastric pains and, in general, to } \\
\text { affect the digestive system, cold, cough, dysmenorrhea, earache, fever, gout, }\end{array}$ & \\
\hline A. moschata Wulfen & $\begin{array}{l}\text { headache, hypertension, insomnia, menopausal disorders, neuralgia, } \\
\text { oliguria, skin inflammations, urinary tract inflammations and vaginitis, } \\
\text { veterinary use }\end{array}$ & [44] \\
\hline A. nobilis L. & Animal parasites, skin wounds and infections & [90] \\
\hline \multirow[t]{2}{*}{ A. santolina $\mathrm{L}$. } & Hypoglycaemia & [56] \\
\hline & $\begin{array}{l}\text { Carminative, antispasmodic, depurative properties, as well as for } \\
\text { stomach-aches and diabetes }\end{array}$ & [56] \\
\hline A. setacea Schwein. & Emmenagogue and stomachic & [71] \\
\hline A. tenuifolia Lam. & Hypercholesterolemia, diabetes, asthma, bronchitis and cough & [71] \\
\hline \multirow[t]{2}{*}{ A. vermicularis Trin. } & Stomachic problems & [71] \\
\hline & Anti-tumour effect & [49] \\
\hline \multirow{3}{*}{ A. wilhelmsii K. Koch. } & Gastrointestinal and pulmonary complaints & [91] \\
\hline & $\begin{array}{l}\text { Diuretic, for abdominal pain, as stomachic and emmenagogues and for } \\
\text { women' sterility, antihaemorrhoidal }\end{array}$ & [71] \\
\hline & $\begin{array}{l}\text { Body- and stomach-ache, blood coagulation, diabetes, hypertension, kidney } \\
\text { stone and constipation }\end{array}$ & [50] \\
\hline
\end{tabular}

\section{Conclusions}

The present review summarizes the existing knowledge on the phytochemistry of the genus Achillea and attempts to bridge, in parallel, the reports on the traditional uses with modern pharmacological data. This survey of the last decade documented 31 Achillea species regarding their phytochemical profiles, including 141 chemical compounds categorized as flavonoids, phenolic and quinic acid derivatives, sesquiterpene lactones and others. Furthermore, 24 species were discussed for their traditional use.

Achillea spp. have been used around the world in folkloric medicine for spasmodic, gastrointestinal and hepato-biliary disorders, haemorrhages, pneumonia, rheumatic pain, wounds healing; as diuretic, anti-inflammatory and antipyretic agents; and to treat menstrual and gynaecologic abnormalities. Some of the reported and validated pharmacological 
activities (in vitro and in vivo) are antioxidant, anti-inflammatory, analgesic, antipyretic, antidiabetic, antibacterial, anthelmintic, and antihypertensive.

The high activity of these plants could be attributed to the presence of a broad range of bio-active metabolites such as flavonoids, sesquiterpene lactones, phenolic acids, sterols, etc., which have been isolated. It is hoped that this work will enrich the current status for the phytochemical and ethnopharmacological background of the genus and will encourage researchers to continue working and further investigate Achillea spp. and its principal metabolites, especially flavonoids and sesquiterpene lactones, as lead compounds for future drug development, targeting serious human ailments.

Future perspectives include the investigation of its traditional uses for gastrointestinal disorders, menstrual and gynaecologic abnormalities, and especially further in vitro, in vivo, and, importantly, clinical studies should be performed to verify the traditional uses for the most recognized species, i.e., A. milefolium L., as well as for less studied or other uninvestigated medicinal plants.

Supplementary Materials: They are available online at https://www.mdpi.com/article/10.339 0/scipharm89040050/s1. Figure S1. Co-authorship/co-occurrence authors networks; Figure S2. Keyword map based on shared networks; Figure S3. Term map from the titles and abstracts on shared networks.

Author Contributions: Conceptualization and design of the work: C.B.; analysis and interpretation of the data: C.B., M.-E.G., E.-M.T. and H.S.; drafting the manuscript: C.B., M.-E.G. and E.-M.T. All authors have read and agreed to the published version of the manuscript.

Funding: This research received no external funding.

Conflicts of Interest: The authors declare no conflict of interest.

\section{References}

1. Dias, D.A.; Urban, S.; Roessner, U. A historical overview of natural products in drug discovery. Metabolites 2012, 2, 303-336. [CrossRef] [PubMed]

2. Turkmenoglu, F.P.; Agar, O.T.; Akaydin, G.; Hayran, M.; Demirci, B. Characterization of volatile compounds of eleven Achillea species from turkey and biological activities of essential oil and methanol extract of A. hamzaoglui Arabacı \& Budak. Molecules 2015, 20, 11432-11458. [CrossRef] [PubMed]

3. Ehrendorfer, F.; Guo, Y.P. Multidisciplinary studies on Achillea sensu lato (Compositae-Anthemideae): New data on systematics and phylogeography. Willdenowia 2006, 36, 69-87. [CrossRef]

4. Guo, Y.P.; Ehrendorfer, F.; Samuel, R. Phylogeny and systematics of Achillea (Asteraceae-Anthemideae) inferred from the nrITS and plastid trnL-F DNA sequences. Taxon 2004, 53, 657-672. [CrossRef]

5. Botanical Database. Available online: http:/ / plantea.myspecies.info/ (accessed on 1 September 2021).

6. Totelin, L.M.V. Hippocratic Recipes: Oral and Written Transmission of Pharmacological Knowledge in Fifth-and Fourth-Century Greece; Brill: Leiden, The Netherlands, 2009. [CrossRef]

7. Papakosta, K.; Grafakou, M.E.; Barda, C.; Kostopoulos, I.V.; Tsitsilonis, O.; Skaltsa, H. Cytotoxicity and anti-cancer activity of the genus Achillea L. Curr. Med. Chem. 2020, 27, 6910-6925. [CrossRef] [PubMed]

8. Applequist, W.L.; Moerman, D.E. Yarrow (Achillea millefolium L.): A neglected panacea? A review of ethnobotany, bioactivity, and biomedical research. Econ. Bot. 2011, 65, 209. [CrossRef]

9. World Health Organization. Available online: www.who.int (accessed on 1 September 2021).

10. European Medicines Agency. Available online: www.ema.europa.eu (accessed on 1 September 2021).

11. Si, X.T.; Zhang, M.L.; Shi, Q.W.; Kiyota, H. Chemical constituents of the plants in the genus Achillea. Chem. Biodivers. 2006, 3, 1163-1180. [CrossRef]

12. Salehi, B.; Selamoglu, Z.; Sevindik, M.; Fahmy, N.M.; Al-Sayed, E.; El-Shazly, M.; Csupor-Löffler, B.; Csupor, D.; Yazdi, S.E.; Sharifi-Rad, J.; et al. Achillea spp.: A comprehensive review on its ethnobotany, phytochemistry, phytopharmacology and industrial applications. Cell Mol. Biol. 2020, 66, 78-103. [CrossRef]

13. Mohammadhosseini, M.; Sarker, S.D.; Akbarzadeh, A. Chemical composition of the essential oils and extracts of Achillea species and their biological activities: A review. J. Ethnopharmacol. 2017, 6, 257-315. [CrossRef]

14. Saeidnia, S.; Gohari, A.; Mokhber-Dezfuli, N.; Kiuchi, F. A review on phytochemistry and medicinal properties of the genus Achillea. DARU J. Pharm. Sci. 2011, 19, 173-186.

15. Blumenthal, M.; Goldberg, A.; Brinckmann, J. Herbal Medicine, Expanded Commission E monographs; The American Botanical Council: Austin TX, USA, 2000; pp. 419-423. 
16. Kaczorová, D.; Karalija, E.; Dahija, S.; Bešta-Gajević, R.; Parić, A.; Ćavar Zeljković, S. Influence of extraction solvent on the phenolic profile and bioactivity of two Achillea species. Molecules 2021, 26, 1601. [CrossRef]

17. Zhang, Q.; Lu, Z.; Ren, T.; Ge, Y.; Zheng, Y.; Yao, D.; He, X.; Gu, Y.; Shi, Q.; Huo, C. Chemical composition of Achillea alpina. Chem. Nat. Compd. 2014, 50, 534-536. [CrossRef]

18. Lee, H.; Sim, M.; Jeong Da-Eun, K.; Jung, H.; An, B.; Cho, H. Antioxidant and anti-melanogenic activities of compounds isolated from the aerial parts of Achillea alpina L. Chem. Biodivers. 2019, 16, e1900033. [CrossRef] [PubMed]

19. Zhang, Q.; Zhou, Q.; Huo, C.; Wang, Y.; Wu, Y.; Zhang, M.; Li, L.; Jin, S.; Shi, Q.; Gu, Y. Phenolic components of the aerial parts of Achillea alpina. Chem. Nat. Compd. 2019, 55, 337-339. [CrossRef]

20. Dorjsembe, B.; Lee, H.J.; Kim, M.; Dulamjav, B.; Jigjid, T.; Nho, C.W. Achillea asiatica extract and its active compounds induce cutaneous wound healing. J. Ethnopharmacol. 2017, 206, 306-314. [CrossRef] [PubMed]

21. Apel, L.; Lorenz, P.; Urban, S.; Sauer, S.; Spring, O.; Stintzing, F.C.; Kammerer, D.R. Phytochemical characterization of different yarrow species (Achillea sp.) and investigations into their antimicrobial activity. ZNC 2020, 76, 55-65. [CrossRef]

22. Salomon, L.; Lorenz, P.; Bunse, M.; Spring, O.; Stintzing, F.C.; Kammerer, D.R. Comparison of the phenolic compound profile and antioxidant potential of Achillea atrata L. and Achillea millefolium L. Molecules 2021, 26, 1530. [CrossRef] [PubMed]

23. Afshari, M.; Rahimmalek, M.; Miroliaei, M. Variation in polyphenolic profiles, antioxidant and antimicrobial activity of different Achillea species as natural sources of antiglycative compounds. Chem. Biodivers. 2018, 15, 1-15. [CrossRef]

24. Abd-Alla, H.; Shalaby, N.; Hamed, M.; El-Rigal, N.S.; Al-Ghamdi, S.; Bouajila, J. Phytochemical composition, protective and therapeutic effect on gastric ulcer and a-amylase inhibitory activity of Achillea biebersteinii Afan. Arch. Pharm. Res. 2015, 39, 10-20. [CrossRef] [PubMed]

25. Şabanoğlu, S.; Gökbulut, A.; Altun, M.L. Characterization of phenolic compounds, total phenolic content and antioxidant activity of three Achillea species. J. Res. Pharm. 2019, 23, 567-576. [CrossRef]

26. Gaweł-Bęben, K.; Strzępek-Gomółka, M.; Czop, M.; Sakipova, Z.; Głowniak, K.; Kukula-Koch, W. Achillea millefolium L. and Achillea biebersteinii Afan. hydroglycolic extracts-bioactive ingredients for cosmetic use. Molecules 2020, 24, 3368. [CrossRef]

27. Agar, O.T.; Dikmen, M.; Ozturk, N.; Yilmaz, M.A.; Temel, H.; Turkmenoglu, F.P. Comparative studies on phenolic composition, antioxidant, wound healing and cytotoxic activities of selected Achillea L. species growing in turkey. Molecules 2015, 20, 1797618000. [CrossRef] [PubMed]

28. Bina, F.; Daglia, M.; Santarcangelo, C. Phytochemical profiling and ameliorative effects of Achillea cretica L. on rat model of endometriosis. J. Ethnopharmacol. 2020, 254, 112747. [CrossRef]

29. Benedec, D.; Vlase, L.; Oniga, I.; Mot, A.C.; Damian, G.; Hanganu, D.; Duma, M.; Silaghi-Dumitrescu, R. Polyphenolic composition, antioxidant and antibacterial activities for two Romanian subspecies of Achillea distans Waldst. et Kit. ex Willd. Molecules 2013, 18, 8725-8739. [CrossRef]

30. Awad, B.M.; Habib, E.S.; Ibrahim, A.K.; Wanas, A.S.; Radwan, M.M.; Helal, M.A.; ElSohly, M.A.; Ahmed, S.A. Cytotoxic activity evaluation and molecular docking study of phenolic derivatives from Achillea fragrantissima (Forssk.) growing in Egypt. Med. Chem. Res. 2017, 26, 2065-2073. [CrossRef]

31. Ezzat, S.M.; Salama, M.M. A new $\alpha$-glucosidase inhibitor from Achillea fragrantissima (Forssk). Sch. Bip. growing in Egypt. Nat. Prod. Res. 2014, 28, 812-881. [CrossRef]

32. Skafa, J.; Hamarshehb, O.; Berningera, M.; Balasubramanianc, S.; Oelschlaegerc, T.; Holzgrabea, U. Improving anti-trypanosomal activity of alkamides isolated from Achillea fragrantissima. Fitoterapia 2018, 125, 191-198. [CrossRef] [PubMed]

33. Abd El-Fattah, A.; Ali, S.; Aly, H.; Abd-Alla, H.; Shalaby, N.; Saleh, M. Therapeutic potential of Achillea fragrantissima extracts in amelioration of high-fat diet and low dose streptozotocin diabetic rats. J. Altern. Complement. Med. 2018, 7, 115-130. [CrossRef]

34. Taşkın, D.; Alkaya, D.B.; Dölen, E. Analysis of natural dyestuffs in Achillea grandifolia Friv. using HPLC-DAD and Q-TOF LC/MS. NISCAIR-CSIR 2017, 16, 83-88.

35. Venditti, A.; Guarcini, L.; Bianco, A.; Rosselli, S.; Bruno, M.; Senatore, F. Phytochemical analysis of Achillea ligustica All. from Lipari Island (Aeolian Islands). Nat. Prod. Res. 2015, 30, 912-919. [CrossRef]

36. Taşkın, T.; Taşkın, D.; Rayaman, E.; Dikpınar, T.; Süzgeç-Selçuk, S.; Arabacı, T. Characterization of the biological activity and phenolics in Achillea lycaonica. Anal. Lett. 2018, 51, 33-48. [CrossRef]

37. Taskin, T.; Dogan, M.; Arabaci, T. Bioassay-guided isolation and antiproliferative efficacy of extract loaded in chitosan nanoparticles and LC-QTOF-MS/MS analysis of Achillea magnifica. S. Afr. J. Bot. 2020, 133, 236-244. [CrossRef]

38. Sevindik, H.G.; Guvenalp, Z.; Yerdelen, K.O.; Yuca, H. The discovery of potential anticholinesterase compounds from Achillea millefolium L. Ind. Crops Prod. 2015, 76, 873-879. [CrossRef]

39. Huo, C.-H.; Li, Y.; Zhang, M.-L.; Wang, Y.-F.; Zhang, Q.; Qin, F.; Shi, Q.-W.; Kiyota, H. Cytotoxic flavonoids from the flowers of Achillea millefolium L. Chem. Nat. Compd. 2013, 48, 958-962. [CrossRef]

40. Dias, M.I.; Barros, L.; Dueñas, M.; Pereira, E.; Carvalho, A.M.; Alves, R.C.; Oliveira, M.B.P.P.; Santos-Buelga, C.; Ferreira, I.C.F.R Chemical composition of wild and commercial Achillea millefolium L. and bioactivity of the methanolic extract, infusion and decoction. Food Chem. 2013, 141, 4152-4160. [CrossRef]

41. Bhat, M.H.; Bhat, K.A.; Prabha, S.; Hamid, A. Antioxidant and cytotoxic activities of Achillea millefolium from Kashmir. JAIR 2014, 2, 487-491.

42. Taşkın, T.; Güler, E.M.; Şentürk, S.; Çelik, D.D.; Arabacı, T.; Gürer, Ü.S. Cytotoxic activity-guided isolation from Achillea monocephala, and biological activities of its different extracts. Compd. J. 2020, 8, 7-14. [CrossRef] 
43. Vitalini, S.; Madeo, M.; Tava, A.; Iriti, M.; Vallone, L.; Avato, P.; Argentieri, M.P. Chemical profile, antioxidant and antibacterial activities of Achillea moschata Wulfen, an endemic species from the alps. Molecules 2016, 21, 1-15. [CrossRef]

44. Argentieri, M.P.; Madeo, M.; Avato, P.; Iriti, M.; Vitalini, S. Polyphenol content and bioactivity of Achillea moschata from the Italian and Swiss Alps. Z. Für Nat. C 2020, 75, 57-64. [CrossRef]

45. Taşkın, D.; Taşkın, T.; Rayamanc, E. Phenolic composition and biological properties of Achillea nobilis L. subsp. neilreichii (Kerner) Formanek. Ind. Crops Prod. 2018, 111, 555-562. [CrossRef]

46. Gharibi, S.; Tabatabaei, B.; Saeidia, G.; Talebib, M.; Matkowskic, A. The effect of drought stress on polyphenolic compounds and expression of flavonoid biosynthesis related genes in Achillea pachycephala Rech. Phytochemistry 2019, 162, 90-98. [CrossRef] [PubMed]

47. Benedec, D.; Hanganu, D.; Oniga, I.; Filip, L.; Bischin, C.; Silaghi-Dumitrescu, R.; Tiperciuc, B.; Vlase, L. Achillea schurii Flowers: Chemical, antioxidant, and antimicrobial investigations. Molecules 2016, 21, 1050. [CrossRef] [PubMed]

48. Moradkhani, S.; Kobarfard, F.; Ayatollahi, S.A.M. Phytochemical investigations on chemical constituents of Achillea tenuifolia Lam. IJPR 2014, 13, 1049-1054. [PubMed]

49. Taskin, T.; Balkan, I.; Tankin, D.; Dogan, A. Characterization of phenolic constituents and pharmacological activity of Achillea vermiculari. IJPS 2019, 81, 293-301. [CrossRef]

50. Khazneh, E.; Hribová, P.; Hošek, J.; Suchý, P.; Kollár, P.; Pražanová, G.; Muselík, J.; Hanaková, Z.; Václavík, J.; Miłek, M.; et al. The chemical composition of Achillea wilhelmsii C. Koch and its desirable effects on hyperglycemia, inflammatory mediators and hypercholesterolemia as risk factors for cardiometabolic disease. Molecules 2016, 21, 404. [CrossRef]

51. Serino, E.; Chahardoli, A.; Badolati, N.; Sirignano, C.; Jalilian, F.; Mojarrab, M.; Farhangi, Z.; Rigano, D.; Stornaiuolo, M.; Shokoohinia, Y.; et al. Salvigenin, a trimethoxylated flavone from Achillea Wilhelmsii C. Koch, exerts combined lipid-lowering and mitochondrial stimulatory effects. Antioxidants 2021, 10, 1042. [CrossRef]

52. Valant-Vetschera, K.M.; Wollenweber, E. Comparative analysis of leaf exudate flavonoids in Achillea subsect. Filipendulinae. Biochem. Syst. Ecol. 1996, 24, 435-446. [CrossRef]

53. Wollenweber, E.; Valant-Vetschera, K.; Ivanceva, S.; Kuzmanov, B. Flavonoid aglycones from the leaf surfaces of some Achillea species. Phytochemistry 1987, 26, 181-182. [CrossRef]

54. Ivanceva, S.; Kuzmanov, B. Flavonoids in genus Achillea, A. nobilis group. Bull Liais. Groupe Polyphén. 1986, 13, 576-579.

55. Ivanceva, S.; Kuzmanov, B. Epicuticular flavonoids in Achillea sect. Filipendulinae; A comparative study. Farmacija 1990, 40, $20-24$.

56. Nemeth, E.; Bernath, J. Biological activities of yarrow species (Achillea spp.). Curr. Pharm. Des. 2008, 14, 3151-3167. [CrossRef] [PubMed]

57. Sun, S.-W.; Wang, R.-R.; Sun, X.-Y.; Fan, J.-H.; Qi, H.; Liu, Y.; Qin, G.-Q.; Wang, W. Identification of transient receptor potential vanilloid 3 antagonists from Achillea alpina L. and separation by liquid-liquid-refining extraction and high-speed counter-current chromatography. Molecules 2020, 25, 2025. [CrossRef] [PubMed]

58. Taşkın, T.; Özakpınar, Ö.B.; Gürbüz, B.; Uras FGürer, U.S.; Bitiş, L. Identification of phenolic compounds and evaluation of antioxidant, antimicrobial and cytotoxic effects of the endemic Achillea multifida. IJTK 2016, 15, 594-603.

59. Mohamed, A.E.-H.H.; Mohamed, N.S.; Hamed, A.R.; Hegazy, M.-E.F. Anti-inflammatory activity of highly oxygenated terpenoids from Achillea biebersteinii Afan. Z. Für Nat. C 2016, 71, 429-432. [CrossRef]

60. Trifunovic, S.; Isaković, A.M.; Isaković, A.; Vučković, I.; Mandić, B.; Novaković, M.; Vajs, V.; Milosavljević, S.; Trajković, V. Isolation, characterization, and in vitro cytotoxicity of new sesquiterpenoids from Achillea clavennae. Planta Med. 2014, 80, 297-305. [CrossRef]

61. Hichri, F.; Znatia, M.; Janneta, H.B.; Bouajilac, J. A new sesquiterpene lactone and secoguaianolides from Achillea cretica L. growing in Tunisia. Ind. Crops Prod. 2015, 77, 735-740. [CrossRef]

62. Saikali, M.; Ghantous, A.; Halawi, R.; Talhouk, S.N.; Saliba, N.A.; Darwiche, N. Sesquiterpene lactones isolated from indigenous Middle Eastern plants inhibit tumor promoter-induced transformation of JB6 cells. BMC Complement. Altern. Med. 2012, 12, 89. [CrossRef]

63. Tohme, R.; Al Aaraj, L.; Ghaddar, T.; Gali-Muhtasib, H.; Saliba, N.A.; Darwiche, N. Differential growth inhibitory Eeffects of highly oxygenated guaianolides isolated from the Middle Eastern indigenous plant Achillea falcata in HCT-116 colorectal cancer cells. Molecules 2013, 18, 8275-8288. [CrossRef]

64. Li, Y.; Ni, Z.-Y.; Zhu, M.-C.; Zhang, K.; Wu, Y.-B.; Dong, M.; Shi, Q.-W.; Huo, C.-H.; Sauriol, F.; Kiyota, H.; et al. Millifolides A-C. New 1,10-Seco-guaianolides from the Flowers of Achillea millefolium. Z. Für Nat. B 2012, 67, 438-446. [CrossRef]

65. Li, Y.; Zhang, M.-L.; Cong, B.; Wang, S.-M.; Dong, M.; Sauriol, F.; Huo, C.-H.; Shi, Q.-W.; Gu, Y.-C.; Kiyota, H. Achillnin A, a Cytotoxic Guaianolide from Flower of yarrow, Achillea millefolium. Biosci. Biotechnol. Biochem. 2011, 75, 1554-1556. [CrossRef]

66. Li, Y.; Zhu, M.C.; Zhang, M.L.; Wang, Y.F.; Dong, M.; Shi, Q.W.; Huo CHSauriol, F.; Kiyota, H.; Gu, Y.-G.; Cong, B. Achillinin B and C, new sesquiterpene dimers isolated from Achillea millefolium. Tetr. Lett. 2012, 53, 2601-2603. [CrossRef]

67. Arias-Durán, L.; Estrada-Soto, S.; Hernández-Morales, M.; Chávez-Silva, F.; Navarrete-Vázquez, G.; León-Rivera, I.; Perea-Arango, I.; Villalobos-Molina, R.; Ibarra-Barajas, M. Tracheal relaxation through calcium channel blockade of Achillea millefolium hexanic extract and its main bioactive compounds. J. Ethnopharmacol. 2020, 253, 112643. [CrossRef] [PubMed]

68. Althaus, J.B.; Kaiser, M.; Brun, R.; Schmidt, T.J. Antiprotozoal activity of Achillea ptarmica (Asteraceae) and its main alkamide constituents. Molecules 2014, 19, 6428-6438. [CrossRef] [PubMed] 
69. Liu, J.; Wang, D.; He, L.; Mao, Q.; Hu, X. A new lignan and a new terpenoid from Achillea millefolium L. Phytochem. Lett. 2017, 22, 247-250. [CrossRef]

70. Sezik, E.; Yesilada, E.; Honda, G. Traditional medicine in Turkey X. Folk medicine in Central Anatolia. J. Ethnopharmacol. 2001, 75, 95-115. [CrossRef]

71. Altundag, E.; Ozturk, M. Ethnomedicinal studies on the plant resources of east Anatolia, Turkey. Procedia Soc. Behav. Sci. 2011, 19, 756-777. [CrossRef]

72. Ristić, M.; Soković, M.; Grubišić, D.; Kovacević, N. Chemical analysis and antifungal activity of the essential oil of Achillea atrata L. J. Essent. Oil Res. 2004, 16, 75-78. [CrossRef]

73. Ghasemi Pirbalouti, A.; Hossayni, I.; Shirmardi, H.A. Essential oil variation, antioxidant and antibacterial activity of mountain fennel (Zaravschanica membranacea (Boiss.) M. Pimen.). Ind. Crops Prod. 2013, 50, 443-448. [CrossRef]

74. Naghibi, F.; Esmaeili, S.; Malekmohammadi, M.; Hassanpour, A.; Mosaddegh, M. Ethnobotanical survey of medicinal plants used traditionally in two villages of Hamedan, Iran. Res. J. Pharmacogn. 2014, 1, 7-14.

75. Saric-Kundalić, B.; Fritz, E.; Dobeš, C.; Saukel, J. Traditional medicine in the pristine village of Prokoško lake on Vranica mountain, Bosnia and Herzegovina. Sci. Pharm. 2010, 78, 275-290. [CrossRef]

76. Saric-Kundalic, B.; Dobes, C.; Klatte-Asselmeyer, V.; Saukel, J. Ethnobotanical study on medicinal use of wild and cultivated plants in middle, south and west Bosnia and Herzegovina. J. Ethnopharmacol. 2010, 131, 33-55. [CrossRef]

77. Skocibusić, M.; Bezić, N.; Dunkić, V.; Radonić, A. Antibacterial activity of Achillea clavennae essential oil against respiratory tract pathogens. Fitoterapia 2004, 75, 733-736. [CrossRef] [PubMed]

78. Ghantous, A.; Nasser, N.; Saab, I.; Darwiche, N.; Saliba, N.A. Structure-activity relationship of seco-tanapartholides isolated from Achillea falcata for inhibition of HaCaT cell growth. Eur. J. Med. Chem. 2009, 44, 3794-3797. [CrossRef] [PubMed]

79. Aminkhani, A.; Sharifi, S.; Ekhtiyari, S. Achillea filipendulina Lam.: Chemical constituents and antimicrobial activities of essential oil of stem, leaf, and flower. Chem. Biodivers. 2020, 17, e2000133. [CrossRef] [PubMed]

80. Choucry, M.A. Chemical composition and anticancer activity of Achillea fragrantissima (Forssk.) Sch. Bip. (Asteraceae) essential oil from Egypt. J. Pharmacogn. Phytother. 2017, 9, 1-5. [CrossRef]

81. Elmann, A.; Mordechay, S.; Erlank, H.; Telerman, A.; Rindner, M.; Ofir, R. Anti-neuroinflammatory effects of the extract of Achillea fragrantissima. BMC Complement. Altern. Med. 2011, 1, 98. [CrossRef]

82. Freires, I.A.; Denny, C.; Benso, B.; De Alencar, S.M.; Rosalen, P.L. Antibacterial activity of essential oils and their isolated constituents against cariogenic bacteria: A systematic review. Molecules 2015, 20, 7329-7358. [CrossRef]

83. Maggi, F.; Bramucci, M.; Cecchini, C.; Coman, M.M.; Cresci, A.; Cristalli, G.; Lupidi, G.; Papa, F.; Quassinti, L.; Sagratini, G.; et al. Composition and biological activity of essential oil of Achillea ligustica All. (Asteraceae) naturalized in central Italy: Ideal candidate for anti-cariogenic formulations. Fitoterapia 2009, 80, 313-319. [CrossRef]

84. Lin, L.-T.; Liu, L.-T.; Chiang, L.-C.; Lin, C.-C. In vitro anti-hepatoma activity of fifteen natural medicines from Canada. Phytother. Res. 2002, 16, 440-444. [CrossRef]

85. Shikov, A.N.; Pozharitskaya, O.N.; Makarov, V.G.; Wagner, H.; Verpoorte, R.; Heinrich, M. Medicinal plants of the Russian pharmacopoeia; their history and applications. J. Ethnopharmacol. 2014, 146, 481-536. [CrossRef]

86. Miraldi, E.; Ferri, S.; Mostaghimi, V. Botanical drugs and preparations in the traditional medicine of West Azerbaijan (Iran). J Ethnopharmacol. 2001, 75, 77-87. [CrossRef]

87. Sharma, P.K.; Chauhan, N.S.; Lal, B. Observations on the traditional phytotherapy among the inhabitants of Parvati valley in western Himalaya, India. J. Ethnopharmacol. 2004, 92, 167-176. [CrossRef] [PubMed]

88. Sayed, A.; Bano, H. Brinjasif (Achillea millefolium Linn): An efficacious unani medicine. Int. J. Herb. Med. 2018, 6, 25-28.

89. Demirci, B.; Baser, K.H.C.; Aytac, Z.; Khan, S.I.; Jacob, M.R.; Tabanca, N. Comparative study of three Achillea essential oils from eastern part of Turkey and their biological activities. Rec. Nat. Prod. 2018, 12, 195-200. [CrossRef]

90. Ghani, A.; Azizi, M.; Hassanzadeh-Khayyat, M.; Pahlavanpour, A.A. Comparison of chemical composition of Achillea eriophora and A. wilhelmsii grown in wild and cultivated conditions in Iran. J. Essent. Oil Bear. Plants 2011, 14, 617-624. [CrossRef]

91. Maffei, M.; Mucciarelli, M.; Scannerini, S. Essential oils from Achillea species of different geographic origin. Biochem. Syst. Ecol. 1994, 22, 679-687. [CrossRef]

92. Kizil, M.; Kizil, G.; Yavuz, M.; ÇeKen, B. Protective activity of ethanol extract of three Achillea species against lipid peroxidation, protein oxidation and DNA damage in vitro. Acta Aliment. 2010, 39, 457-470. [CrossRef]

93. Barış, D.; Kızıl, M.; Aytekin, C.; Kızıl, G.; Yavuz, M.; Çeken, B.; Selçuk Ertekin, A. In vitro antimicrobial and antioxidant activity of ethanol extract of three Hypericum and three Achillea species from Turkey. Int. J. Food Prop. 2011, 14, 339-355. [CrossRef]

94. Qi, H.; Shi, Y.; Wu, H.; Niu, C.; Sun, X.; Wang, K. Inhibition of temperature-sensitive TRPV3 channel by two natural isochlorogenic acid isomers for alleviation of dermatitis and chronic pruritus. Acta Pharm. Sin. B 2021. [CrossRef]

95. Aljančić, I.; Vajs, V.; Menković, N.; Karadžić, I.; Juranić, N.; Milosavljević, S.; Macura, S. Flavones and sesquiterpene lactones from Achillea atrata subsp. multifida: Antimicrobial activity. J. Nat. Prod. 1999, 62, 909-911. [CrossRef]

96. Zengin, G.; Aktumsek, A.; Ceylan, R.; Uysal, S.; Mocan, A.; Guler, G.O. Shedding light on the biological and chemical fingerprints of three Achillea species (A. biebersteinii, A. millefolium and A. teretifolia). Food Funct. 2017, 8, 1152-1165. [CrossRef]

97. Akkol, E.K.; Koca, U.; Pesin, I.; Yilmazer, D. Evaluation of the wound healing potential of Achillea biebersteinii Afan. (Asteraceae) by in vivo excision and incision models. Evid. Based Complement. Altern. Med. 2011, 2011, 474026. [CrossRef] [PubMed] 
98. Abbas, M.A.; Jaffal, S.M.; Al-Najjar, B.O. Analgesic and anxiolytic activities of Achillea biebersteinii: Evidence for the involvement of GABAergic systems. Orient. J. Chem. 2019, 35, 1433-1442. [CrossRef]

99. Ertaş, A.; Boğa, M.; Haşimi, N.; Yeşil, Y.; Gören, A.C.; Topçu, G.; Kolak, U. Antioxidant, anticholinesterase, and antimicrobial activities and fatty acid constituents of Achillea cappadocica Hausskn. et Bornm. Turk. J. Chem. 2014, 38, 592-599. [CrossRef]

100. Giorgi, A.; Bombelli, R.; Luini, A.; Speranza, G.; Cosentino, M.; Lecchini, S.; Cocucci, M. Antioxidant and cytoprotective properties of infusions from leaves and inflorescences of Achillea collina Becker ex Rchb. Phytother. Res. 2009, 23, 540-545. [CrossRef] [PubMed]

101. Gherase, F.; Pavelescu, M.D.; Stănescu, U.; Grigorescu, E. The experimental evaluation regarding analgesic activity of some extracts isolated from Achillea collina J. Becker ex Reichenb. Rev. Med. Chir. Soc. Med. Nat. Iasi 2002, 106, 801-805. [CrossRef] [PubMed]

102. Stojanović, G.; Radulović, N.; Hashimoto, T.; Palić, R. In vitro antimicrobial activity of extracts of four Achillea species: The composition of Achillea clavennae L. (Asteraceae) extract. J. Ethnopharmacol. 2005, 101, 185-190. [CrossRef] [PubMed]

103. Karaalp, C.; Yurtman, A.N.; Yavasoglu, N.U.K. Evaluation of antimicrobial properties of Achillea L. flower head extracts. Pharm. Biol. 2009, 47, 86-91. [CrossRef]

104. Hammad, M.H.; Carmen Litescu, S.; Matar, S.A.; Al-Jaber, I.H.; Afifi, F.U. Biological activities of the hydro-alcoholic and aqueous extracts of Achillea falcata L. (Asteraceae) grown in Jordan. EJMP 2013, 4, 259-270. [CrossRef]

105. Gharibi, S.; Sayed Tabatabaei, B.E.; Saeidi, G. Comparison of essential oil composition, flavonoid content and antioxidant activity in eight Achillea Species. J. Essent. Oil Bear. Plants 2015, 18, 1382-1394. [CrossRef]

106. Abdel-Rahman, R.F.; Alqasoumi, S.I.; El-Desoky, A.H.; Soliman, G.A.; Paré, P.W.; Hegazy, M.-E. Evaluation of the antiinflammatory, analgesic and anti-ulcerogenic potentials of Achillea fragrantissima (Forssk.). S. Afr. J. Bot. 2015, 98, 122-127. [CrossRef]

107. Tuberoso, C.I.; Montoro, P.; Piacente, S.; Corona, G.; Deiana, M.; Dessì, M.A.; Pizza, C.; Cabras, P. Flavonoid characterization and antioxidant activity of hydroalcoholic extracts from Achillea ligustica All. J. Pharm. Biomed. Anal. 2009, 50, 440-448. [CrossRef]

108. Bader, A.; Martini, F.; Schinella, G.R.; Rios, J.L.; Prieto, J.M. Modulation of COX-1, 5-, 12- and 15-LOX by popular herbal remedies used in southern Italy against psoriasis and other skin diseases. Phytother. Res. 2015, 29, 108-113. [CrossRef] [PubMed]

109. Conforti, F.; Loizzo, M.R.; Statti, G.A.; Menichini, F. Comparative radical scavenging and antidiabetic activities of methanolic extract and fractions from Achillea ligustica ALL. Biol. Pharm. Bull. 2005, 28, 1791-1794. [CrossRef] [PubMed]

110. Tadić, V.; Arsić, I.; Zvezdanović, J.; Zugić, A.; Cvetković, D.; Pavkov, S. The estimation of the traditionally used yarrow (Achillea millefolium L. Asteraceae) oil extracts with anti-inflamatory potential in topical application. J. Ethnopharmacol. 2017, 199, 138-148. [CrossRef]

111. Benedek, B.; Kopp, B. Achillea millefolium L. s.l. revisited: Recent findings confirm the traditional use. Wien. Med. Wochenschr. 2007, 157, 312-314. [CrossRef]

112. Baretta, I.P.; Felizardo, R.A.; Bimbato, V.F.; dos Santos, M.G.J.; Kassuya, C.A.L.; Gasparotto Junior, A.; da Silva, C.R.; de Oliveira, A.M.; Ferreira, J.; Andreatini, R. Anxiolytic-like effects of acute and chronic treatment with Achillea millefolium L. extract. J. Ethnopharmacol. 2012, 140, 46-54. [CrossRef]

113. Kasabri, V.; Afifi, F.U.; Hamdan, I. In vitro and in vivo acute antihyperglycemic effects of five selected indigenous plants from Jordan used in traditional medicine. J. Ethnopharmacol. 2011, 133, 888-896. [CrossRef]

114. Yazdanparast, R.; Ardestani, A.; Jamshidi, S. Experimental diabetes treated with Achillea santolina: Effect on pancreatic oxidative parameters. J. Ethnopharmacol. 2007, 112, 13-18. [CrossRef]

115. Zitterl-Eglseer, K.; Jurenitsch, J.; Korhammer, S.; Haslinger, E.; Sosa, S.; Della Loggia, R.; Kubelka, W.; Franz, C. Entzündungshemmende Sesquiterpenlactone von Achillea setacea. Sesquiterpenelactones of Achillea setacea with antiphlogistic activity. Planta Med. 1991, 57, 444-446. [CrossRef]

116. Bagheri, Y.; Fathi, E.; Maghoul, A.; Moshtagh, S.; Mokhtari, K.; Abdollahpour, A.; Montazersaheb, S.; Bagheri, A. Effects of Achillea tenuifolia Lam. hydro-alcoholic extract on anxiety-like behavior and reproductive parameters in rat model of chronic restraint stress. Hum. Exp. Toxicol. 2021, 25, 9603271211026723. [CrossRef]

117. Hamzeloo-Moghadam, M.; Khalaj, A.; Malekmohammadi, M.; Mosaddegh, M. Achillea vermicularis a medicinal plant from Iranian Traditional Medicine induces apoptosis in MCF-7 cells. RJP 2015, 2, 1-5.

118. Bashi, D.S.; Bazzaz, B.S.F.; Sahebkar, A.; Karimkhani, M.M.; Ahmadi, A. Investigation of optimal extraction, antioxidant, and antimicrobial activities of Achillea biebersteinii and A. wilhelmsii. Pharm. Biol. 2012, 50, 1168-1176. [CrossRef]

119. Amjad, L.; Mohammadi-Sichani, M.; Mohammadi-Kamalabadi, M. Potential activity of the Achillea wilhelmsii leaves on bacteria. Int. J. Biosci. Biochem. Bioinform. 2011, 1, 216-218. [CrossRef]

120. Niazmand, S.; Khooshnood, E.; Derakhshan, M. Effects of Achillea wilhelmsii on rat's gastric acid output at basal, vagotomized, and vagal-stimulated conditions. Pharmacogn. Mag. 2010, 6, 282-285. [CrossRef] [PubMed]

121. Niaz, A.; Syed Wadood, A.S.; Ghayour, A.; Ismail, S.; Mohammad, S.; Muhammad, J.; Waqar, A. Acute toxicity and antispasmodic activities of Achillea wilhelmsii C. Koch. Pak. J. Pharm. Sci. 2014, 27, 309-315.

122. Mohammadmehdi, H.-T.; Hassanpour-Fard, M.; Ahani, A.; Hosseini, M. Anti-Nociceptive activity of Achillea wilhelmsii ethanolic extract in mice. J. Complement. Med. Altern. Healthc. 2018, 6, 555685. [CrossRef] 